\title{
New tests of local Lorentz invariance of gravity with small-eccentricity binary pulsars
}

\author{
Lijing Shao ${ }^{1,2}$ and Norbert Wex ${ }^{1}$ \\ ${ }^{1}$ Max-Planck-Institut für Radioastronomie, Auf dem Hügel 69, 53121 Bonn, Germany \\ ${ }^{2}$ School of Physics, Peking University, Beijing 100871, China \\ E-mail: 1shao@pku.edu.cn, wex@mpifr-bonn.mpg.de
}

\begin{abstract}
.
Some alternative gravity theories allow the Universal matter distribution to single out the existence of a preferred frame, which breaks the symmetry of local Lorentz invariance (LLI) for the gravitational interaction. In the post-Newtonian parametrization of semi-conservative gravity theories, LLI violation is characterized by two parameters, $\alpha_{1}$ and $\alpha_{2}$. In binary pulsars the isotropic violation of Lorentz invariance in the gravitational sector should lead to characteristic preferred frame effects (PFEs) in the orbital dynamics, if the barycenter of the binary is moving relative to the preferred frame with a velocity $\mathbf{w}$.

For small-eccentricity binaries, the effects induced by $\hat{\alpha}_{1}$ and $\hat{\alpha}_{2}$ (the hat indicates possible modifications by strong-field effects) decouple, and can therefore be tested independently. We use recent timing results of two compact pulsar-white dwarf binaries with known 3-dimensional velocity, PSRs J1012+5307 and J1738+0333, to constrain PFEs for strongly self-gravitating bodies, by assuming the isotropic cosmic microwave background to single out a preferred frame. The time derivative of the projected semimajor axis is used to constrain a precession of the orbital plane around $\mathbf{w}$ due to PFEs. From this we derive a limit $\left|\hat{\alpha}_{2}\right|<1.8 \times 10^{-4}$ at $95 \%$ confidence level, which is the most constraining limit for strongly self-gravitating systems up to now, however still three orders of magnitude weaker than the best Solar system limit for the corresponding weak-field parameter $\alpha_{2}$.

Concerning $\hat{\alpha}_{1}$, we propose a new, robust method to constrain this parameter, which avoids the probabilistic considerations inherent in previous methods. This method is based on the fact that a PFE-induced intrinsic eccentricity cannot stay unobserved during a long-term observation due to the significant precession of periastron in binary pulsar with short orbital periods. Our most conservative result, $\hat{\alpha}_{1}=-0.4_{-3.1}^{+3.7} \times 10^{-5}$ at $95 \%$ confidence level from PSR J1738+0333, constitutes a significant improvement compared to current most stringent limits obtained both in Solar system and binary pulsar tests.

We also derive corresponding limits for $\hat{\alpha}_{1}$ and $\hat{\alpha}_{2}$ for a preferred frame that is at rest with respect to our Galaxy, and preferred frames that locally co-move with the rotation of our Galaxy.

These limits will continue to improve significantly with future pulsar timing observations conducted at large radio telescopes.
\end{abstract}

PACS numbers: 04.80.Cc, 11.30.Cp, 97.60.Gb 


\section{Introduction}

Lorentz invariance is one of the most important ingredients inherent in modern theoretical physics, including the standard model of particle physics and general relativity (GR). From a group theoretical viewpoint, it composes of two parts, rotational invariance and boost invariance. Rotational invariance forms a compact group, i.e., the $\mathrm{SO}(3)$ group, which can be probed throughout, while boost invariance forms a noncompact group, hence it, in principle, cannot be tested thoroughly, and deserves more scrutinies. Lorentz invariance is examined in particle physics to high precision [30], while not so well tested in gravitational physics [53], due to the challanges in gravitational precision experiments.

On the other hand, some alternative gravitational theories predict the existence of a preferred frame, which might be singled out by the matter distribution in our Universe or through historical relics of vectorial or tensorial vacuum expectation values, if gravitational interaction is mediated by a vector field or a second tensor field, in addition to the canonical second-rank symmetric tensor field [54]. These theories include vector-metric theories [54, 52, Einstein-Æther theories [22], TeVeS theories [5], and standard model extensions of gravity [3].

In the parametrized post-Newtonian (PPN) formalism, preferred frame effects (PFEs) are characterized by three parameters, $\alpha_{1}, \alpha_{2}$ and $\alpha_{3}$ [54, 52]. Since $\alpha_{3}$ also causes energy-momentum conservation violation, and is well constrained to very high precision (see e.g. [48], where the strong-field version of $\left|\alpha_{3}\right|$ is constrained to be less than $4 \times 10^{-20}$ at $95 \%$ confidence level, by using wide-orbit binary millisecond pulsars), we will not consider it further in this work. Experimental tests on PFEs induced by $\alpha_{1}$ and $\alpha_{2}$ are roughly divided into three catalogues, i.e., geophysical tests, Solar system tests, and pulsar timing tests.

Nordtvedt and Will [43] derived possible experimental indications of a preferred frame for the gravitational interaction in geophysics and orbital motions, e.g., an anomalous 12-hour sidereal tide of the solid Earth, an anomalous yearly variation in the rotational frequency of the Earth, and an anomalous perihelion shift of the planets. By now, the best limit for $\alpha_{1}$ in the Solar system comes from Lunar Laser Ranging $(\mathrm{LLR})$, that places a $95 \%$ confidence limit of $\alpha_{1}=(-0.7 \pm 1.8) \times 10^{-4}$ [39]. For the $\alpha_{2}$ parameter, Nordtvedt [42] used the close alignment of the spin axis of the Sun and the total angular momentum vector of the Solar system to limit $\left|\alpha_{2}\right|<2.4 \times 10^{-7}$, under the assumption that the above two vectors were aligned when the Solar system formed

five billion years ago (note, $\alpha_{2}^{\text {Nordtvedt }}=\frac{1}{2} \alpha_{2}$ ). Damour and Esposito-Farèse developed a method to put tight constraints onto the strong-field counterpart of $\alpha_{1}$, namely $\hat{\alpha}_{1}$, from timing experiments of small-eccentricity binary pulsars [12]. Their calculation shows that the observational eccentricity vector, $\mathbf{e}(t)$, is a vectorial superposition of a "rotating eccentricity" $\mathbf{e}_{R}(t)$ with constant length $e_{R}$, and a fixed "forced eccentricity" $\mathbf{e}_{F}$. From probabilistic consideration, they were able to constrain $\left|\hat{\alpha}_{1}\right|$ to be less than $5.0 \times 10^{-4}$ (90\% C.L.). This limit has been improved by a factor of three in [6], based on 
the small-eccentricity binary pulsar PSR J2317+1439. Wex [50] extended this method in statistically combining multiple systems, by taking care of a potential selection effect when simply picking the system with the most favorable parameter combination. He got a slightly improved result of $\left|\hat{\alpha}_{1}\right|<1.2 \times 10^{-4}$ (95\% C.L.). In section 4 , we extend the statistically dependent method into a robust one which not only avoids involving probabilistic considerations concerning certain unobservable angles, but also gets a new constraint, $\hat{\alpha}_{1}=-0.4_{-3.1}^{+3.7} \times 10^{-5}(95 \%$ C.L. $)$, that surpasses the current best constraints of both weak and strong fields.

For the $\alpha_{2}$ parameter, because of the tight limit of [42], Damour and EspositoFarèse [12] dropped the $\alpha_{2}$ term when calculating binary orbital dynamics. We stress that the limit of [42] is obtained in a weak-field gravitational environment, while in the strong-field regime, like inside a neutron star (NS), $\alpha_{2}$ might take an independent value, significantly different from the Solar system value. In fact, it was discovered that in certain classes of tensor-scalar theories of gravity, large non-perturbative strong-field deviations from GR can occur, through a phenomenon called "spontaneous scalarization" 14. Although tensor-scalar theories of gravity are conservative gravity theories and do not show PFEs, it is natural to assume the possibility of similar non-perturbative effects in gravitational theories with local Lorentz invariance (LLI) violation. Hence, we feel that it is still worth to independently test, in a phenomenological approach, the strong-field counterpart of $\alpha_{2}$, namely $\hat{\alpha}_{2}$, in pulsar binary timing experiments.

Following [12, we calculate the $\hat{\alpha}_{2}$ effect for pulsar binaries, and find that it practically decouples from the $\hat{\alpha}_{1}$ effect for small orbital eccentricities $(e \ll 1)$. The $\hat{\alpha}_{1}$ term tends to polarize the eccentricity vector towards a direction perpendicular to the orbital angular momentum and the binary barycentric velocity with respect to the preferred frame, w [12]. It causes dynamical effects inside the orbital plane. In contrast, the $\hat{\alpha}_{2}$ term imposes a precession of the orbital angular momentum around the direction of $\mathbf{w}$. It causes a change in the orbital inclination angle with respect to the line of sight, $i$. Consequently, $\hat{\alpha}_{2}$ induces a non-vanishing time derivative of the projected semi-major axis.

Wex and Kramer [51] developed a pulsar timing model that includes PFEs, by extending the widely used Damour-Deruelle timing model [10]. Based on this model, they analyzed the time of arrivals (TOAs) of the double pulsar, PSR J0737-3039A/B [7, 37, 31, 32, and jointly limited $\hat{\alpha}_{1}$ and $\hat{\alpha}_{2}$ to be, $-0.5<\hat{\alpha}_{1}<0.3$ and $-0.3<\hat{\alpha}_{2}<0.2$, respectively $\ddagger$ Their analysis utilized two specific aspects of the double pulsar: 1) The measurement of the mass-ratio via the "double-line" nature of the system, and the measurement of the Shapiro delay allowed for a theory-independent determination of the effective gravitating masses of the two pulsars; 2) The large rate of periastron advance, $\dot{\omega}=16.9 \mathrm{deg} \mathrm{yr}^{-1}$ [31], would significantly change the binary orientation with respect to $\ddagger$ They use $\alpha_{1}^{*}$ and $\alpha_{2}^{*}$ instead of $\hat{\alpha}_{1}$ and $\hat{\alpha}_{2}$ in their notation. The limit quoted above assumes the preferred frame to be at rest with respect to the isotropic cosmic microwave background (see the original paper for constraints on other directions). 
a preferred frame within just a few years, leading to distinct long-term periodic effects in the orbital parameters. Consequently, the double pulsar has even the potential to measure $\hat{\alpha}_{1}$ and $\hat{\alpha}_{2}$, if they are non-zero. As emphasized in [51], double NS systems probe different aspects of a violation of LLI in the gravitational sector (interaction between two strongly self-gravitating bodies) from other kinds of binaries, e.g., NS-white dwarf (WD) binaries. Furthermore, from the simulations in [51] one expects that by now the precision of the PFE test with the double pulsar has greatly improved compared to the numbers in [51].

To this point, let us briefly summarize current best limits on the LLI violation in the weak field and strong field.

(i) Weak field

- From LLR [39],

$$
\alpha_{1}=(-0.7 \pm 1.8) \times 10^{-4} \quad(95 \% \text { C.L. }) .
$$

- From the close alignment of the spin of the Sun with the total angular momentum of the Solar system [42,

$$
\left|\alpha_{2}\right|<2.4 \times 10^{-7} \text {. }
$$

One should be aware that this result depends on an assumption about the alignment of the spin of the Sun and the angular momentum of the Solar system right after their formation, five billion years ago. LLR experiments [39] get a weaker limit, $\alpha_{2}=(1.8 \pm 5.0) \times 10^{-5}(95 \%$ C.L. $)$.

(ii) Strong field

- From the population of small-eccentricity NS-WD binaries [50],

$$
\left|\hat{\alpha}_{1}\right|<1.2 \times 10^{-4} \quad(95 \% \text { C.L. }) .
$$

- From a NS-NS system, namely the double pulsar [51,

$$
-0.3<\hat{\alpha}_{2}<0.2 \quad \text { (95\% C.L.) }
$$

In this paper, we derive the full secular dynamical evolution of a pulsar binary system of arbitrary eccentricity, under the influence of both $\hat{\alpha}_{1}$ and $\hat{\alpha}_{2}$. Afterwards, we utilize our analytical results to propose two new methods to constrain $\hat{\alpha}_{1}$ and $\hat{\alpha}_{2}$, respectively. By using Monte Carlo simulations, we are able to get stringent limits from small-eccentricity NS-WD binaries, PSRs J1012+5307 [33, 34] and J1738+0333 [1, 20], with measurement errors properly accounted for.

The paper is organized as follows. In section 2 we derive the orbital dynamics from a generic semi-conservative Lagrangian keeping both the $\hat{\alpha}_{1}$ and the $\hat{\alpha}_{2}$ terms, for arbitrarily eccentric orbits. We find that in the limit of a small eccentricity, these two parameters decouple $-\hat{\alpha}_{1}$ affects the evolution of the eccentricity vector in the orbital plane, while $\hat{\alpha}_{2}$ controls the precession of the orbital angular momentum. We introduce the isotropic cosmic microwave background (CMB) frame as the most important preferred frame, for our subsequent numerical calculations in sections 3 and 4 . In section 3, $\left|\hat{\alpha}_{2}\right|$ is derived to be less than $1.8 \times 10^{-4}$ (95\% C.L.) from timing experiments 
of pulsar binaries PSRs J1012+5307 and J1738+0333. In section 4, we develop a new, robust method to constrain $\hat{\alpha}_{1}$, which overcomes the need of probabilistic considerations inherent in the former methods. The most conservative limit, $\hat{\alpha}_{1}=-0.4_{-3.1}^{+3.7} \times 10^{-5}$ (95\% C.L.), is derived from the PSR J1738+0333 binary system. Section 5 gives the corresponding results on $\hat{\alpha}_{1}$ and $\hat{\alpha}_{2}$ when the Galaxy or the local Galactic rotation are assumed to single out a preferred frame. The limits for these frames of reference are found to be slightly weaker than the ones for the CMB frame. Furthermore, we give a discussion on the strong-field aspects of our tests, discuss future improvements of these two tests, and briefly summarize the results of the paper.

\section{Binary dynamics of the semi-conservative Lagrangian}

We consider the two-body dynamics of a binary system consisting of a pulsar with mass $m_{p}$ and its companion with mass $m_{c}$. In the presence of a preferred reference frame, the orbital motion of such a system is described by a two-body non-boost-invariant Lagrangian [52, 12]

$$
L=L_{\hat{\beta}, \hat{\gamma}}+L_{\hat{\alpha}_{1}}+L_{\hat{\alpha}_{2}}
$$

The Lagrangian (5) consists of $L_{\hat{\beta}, \hat{\gamma}}$, the post-Newtonian (PN) terms from GR and its minimal extensions characterized by the (strong-field) Eddington-Robertson-Schiff parameters, $\hat{\beta}$ and $\hat{\gamma}$,

$$
\begin{aligned}
L_{\hat{\beta}, \hat{\gamma}}= & -m_{p} c^{2} \sqrt{1-\frac{\left(\mathbf{v}_{p}^{0}\right)^{2}}{c^{2}}}-m_{c} c^{2} \sqrt{1-\frac{\left(\mathbf{v}_{c}^{0}\right)^{2}}{c^{2}}}+\frac{\hat{G} m_{p} m_{c}}{r}\left[1+\frac{\left(\mathbf{v}_{p}^{0}\right)^{2}+\left(\mathbf{v}_{c}^{0}\right)^{2}}{2 c^{2}}\right. \\
& \left.-\frac{3\left(\mathbf{v}_{p}^{0} \cdot \mathbf{v}_{c}^{0}\right)}{2 c^{2}}-\frac{\left(\mathbf{n} \cdot \mathbf{v}_{p}^{0}\right)\left(\mathbf{n} \cdot \mathbf{v}_{c}^{0}\right)}{2 c^{2}}+\hat{\gamma} \frac{\left(\mathbf{v}_{p}^{0}-\mathbf{v}_{c}^{0}\right)^{2}}{c^{2}}-(2 \hat{\beta}-1) \frac{\hat{G} M}{2 c^{2} r}\right],
\end{aligned}
$$

and the velocity-dependent, non-boost-invariant terms, related to non-vanishing $\hat{\alpha}_{1}$ and $\hat{\alpha}_{2}$,

$$
\begin{aligned}
L_{\hat{\alpha}_{1}} & =-\hat{\alpha}_{1} \frac{\hat{G} m_{p} m_{c}}{r} \frac{\left(\mathbf{v}_{p}^{0} \cdot \mathbf{v}_{c}^{0}\right)}{2 c^{2}} \\
L_{\hat{\alpha}_{2}} & =\hat{\alpha}_{2} \frac{\hat{G} m_{p} m_{c}}{r} \frac{\left(\mathbf{v}_{p}^{0} \cdot \mathbf{v}_{c}^{0}\right)-\left(\mathbf{n} \cdot \mathbf{v}_{p}^{0}\right)\left(\mathbf{n} \cdot \mathbf{v}_{c}^{0}\right)}{2 c^{2}}
\end{aligned}
$$

where $M \equiv m_{p}+m_{c}, r \equiv|\mathbf{r}|$ is the coordinate separation of two components, $\mathbf{n} \equiv \mathbf{r} / r$, $\mathbf{v}^{0}$ denotes the "absolute" velocity with respect to the preferred frame, and $c$ is the speed of light. In the above Lagrangian, we add a "hat" onto the notations of $\gamma, \beta$, $\alpha_{1}, \alpha_{2}$, and also the gravitational constant $G$, to underline the fact that we are dealing with the PN parameters associated with compact objects, where strong-field effects might contribute to these values, making them different from their counterparts in the weak field. The specific dependence on the strong-field contributions depends on the gravitational theories under consideration. In GR, one finds $\hat{G}=G, \hat{\beta}=\hat{\gamma}=1$, and $\hat{\alpha}_{1}=\hat{\alpha}_{2}=0$. 
The Lagrangian (5) can be obtained from a more generalized, semi-conservative Einstein-Infeld-Hoffmann Lagrangian $\delta$ in the calculations of [52] and [51], by setting $\mathcal{A}_{p}=\mathcal{A}_{c}=1, \mathcal{G}=\hat{G} / G, \mathcal{B} / \mathcal{G}=\frac{1}{3}(2 \hat{\gamma}+1), \mathcal{C} / \mathcal{G}=\frac{1}{7}\left(4 \hat{\gamma}+\hat{\alpha}_{1}-\hat{\alpha}_{2}+3\right), \mathcal{E} / \mathcal{G}=\hat{\alpha}_{2}+1$, $X_{c} \mathcal{D}_{p} / \mathcal{G}^{2}+X_{p} \mathcal{D}_{c} / \mathcal{G}^{2}=2 \hat{\beta}-1$, where $X_{p} \equiv m_{p} / M$ and $X_{c} \equiv m_{c} / M$.

The assumption $\mathcal{A}_{p}=\mathcal{A}_{c}=1$, made in this paper, requires additional justification. These parameters are equal to one at first post-Newtonian order [52], but for strongly self-gravitating bodies, e.g. NSs, they could significantly deviate from one (e.g. in Einstein-Æther theory [19]). The subject of this paper are pulsars with low-mass WD companions, and therefore $\mathcal{A}_{c} \simeq 1$. Concerning $\mathcal{A}_{p}$, it enters the secular changes of the relevant orbital parameters only as addition to the parameters $\hat{\alpha}_{1}$ and $\hat{\alpha}_{2}$ (see [51] for details). In the first case the $\mathcal{A}_{p}$ contribution is multiplied by a factor of $2 X_{c}^{2}$ and in the second case by a factor $X_{c}$. Since $X_{c} \sim 0.1$, for our binary systems, in both cases the $\mathcal{A}_{p}$ contributions are expected to be small with respect to the $\hat{\alpha}_{1}$ and $\hat{\alpha}_{2}$ terms.

Besides the PPN parameters $\beta, \gamma, \alpha_{1}$ and $\alpha_{2}$, semi-conservative theories of gravity could have a non-zero Whitehead term, characterized by $\xi$ [52]. It reflects preferredlocation effects, such as an anisotropy in the local gravitational constant caused by an external gravitational potential. Various well-motivated (fully conservative and semiconservative) gravity theories have $\xi=0$ (for instance, see Table 3 of [53]). Therefore, we will only include $\hat{\beta}, \hat{\gamma}, \hat{\alpha}_{1}$, and $\hat{\alpha}_{2}$ in our following discussion, and ignore a potential strong-field counterpart of the Whitehead term. We note in passing, that for smalleccentricity binaries, the presence of a Whitehead term only changes the $\hat{\alpha}_{2}$ test, which then constrains a combination of $\hat{\alpha}_{2}$ and $\hat{\xi}$. The $\hat{\alpha}_{1}$ test, on the other hand, remains unchanged. This can be readily seen from (8.73) in [52].

\subsection{Orbital dynamics in the presence of PFEs}

Using the Euler-Lagrange equations and the post-Galilean transformations [9], we can get the relative acceleration for a pulsar binary system in the comoving frame, whose center of mass moves relative to the preferred frame with a velocity $\mathbf{w}$,

$$
\ddot{\mathbf{r}} \equiv \ddot{\mathbf{r}}_{p}-\ddot{\mathbf{r}}_{c}=\mathbf{A}^{(\mathrm{N})}+\mathbf{A}^{(\mathrm{PN})} / c^{2}+\mathbf{A}^{(\mathbf{w})} / c^{2},
$$

where $\mathbf{A}^{(\mathrm{N})}$ is the "Newtonian" acceleration, $\mathbf{A}^{(\mathrm{N})}=-\hat{G} M \mathbf{n} / r^{2}, \mathbf{A}^{(\mathrm{PN})} / c^{2}$ is the first $\mathrm{PN}$ acceleration without $\mathbf{w}$-dependent contributions, and $\mathbf{A}^{(\mathbf{w})} / c^{2}$ is the additional acceleration from the motion of the binary system with respect to the preferred frame. For expressions of these accelerations, see [52, 12, 51].

As is well known, the acceleration $\mathbf{A}^{(\mathrm{PN})} / c^{2}$ produces a secular advance of the longitude of periastron,

$$
\dot{\omega}_{\mathrm{PN}}=\frac{3 \mathcal{V}_{O}^{2} \mathcal{F}}{c^{2}\left(1-e^{2}\right)} n_{b},
$$

$\S$ A "semi-conservative" Lagrangian corresponds to a gravity theory that possesses conservation laws for the total energy and momentum. Any theory that is based on an invariant action principle is "semi-conservative" [53]. 


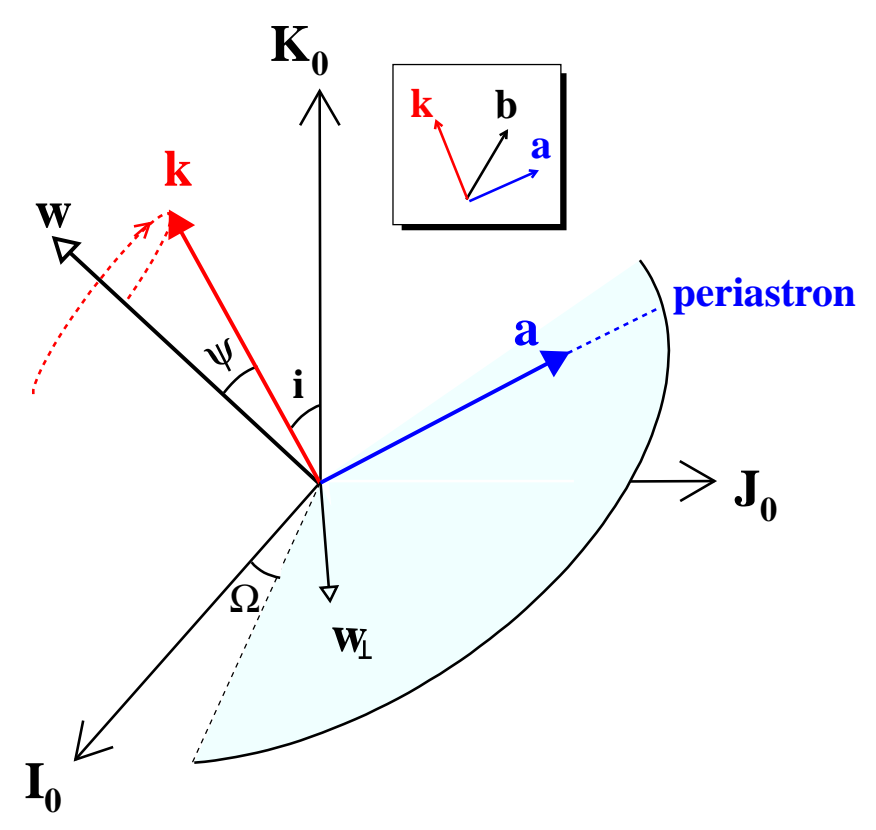

Figure 1. Illustration of the geometry of the binary system, and the notation used in the paper. The coordinate system $\left(\mathbf{I}_{0}, \mathbf{J}_{0}, \mathbf{K}_{0}\right)$ corresponds to $\left(\vec{I}_{0}, \vec{J}_{0}, \vec{K}_{0}\right)$ in figure 1 or $\left(\mathbf{I}_{0}, \mathbf{J}_{0}, \mathbf{K}_{0}\right)$ in the text of [18, and the coordinate system $(\mathbf{a}, \mathbf{b}, \mathbf{k})$ corresponds to $(\mathbf{a}, \mathbf{b}, \mathbf{c})$ in $[12$. $\mathbf{w}$ is the velocity of the binary system with respect to the preferred frame, while $\mathbf{w}_{\perp}$ is its projection into the orbital plane.

where

$$
\begin{aligned}
& \mathcal{V}_{O} \equiv\left(\hat{G} M n_{b}\right)^{1 / 3}, \\
& \mathcal{F} \equiv \frac{1}{3}(2+2 \hat{\gamma}-\hat{\beta})+\frac{1}{6}\left(2 \hat{\alpha}_{1}-\hat{\alpha}_{2}\right) X_{p} X_{c},
\end{aligned}
$$

and $n_{b}=2 \pi / P_{b}$ is the orbital frequency of the binary system. The "characteristic" velocities in $\mathrm{GR}, \mathcal{V}_{O}^{(\mathrm{GR})}$, for pulsar binaries PSRs J1012+5307 and J1738+0333, are given in table 1, In $\mathrm{GR}, \mathcal{F}=1$. Because $\mathbf{A}^{(\mathrm{PN})} / c^{2}$ lies in the orbital plane, it has no effect on the longitude of the ascending node $\Omega$, and the orbital inclination angle $i$ (see figure 1 for illustration of these angles). In addition, $\mathbf{A}^{(\mathrm{PN})} / c^{2}$ is verified to have no effect on $e$, the length of the eccentricity vector, and $a$, the semi-major axis of the relative orbit (cf. (15) below, with $\mathbf{w}=0$ )

As for the acceleration from PFEs, $\mathbf{A}^{(\mathbf{w})} / c^{2}$, Damour and Esposito-Farèse [12] worked out the influence of $\hat{\alpha}_{1}$-related terms on the orbital evolution. After averaging over one orbital period, they found for changes in the semi-major axis $a, \mathbf{l} \equiv \sqrt{1-e^{2}} \mathbf{k}$,

$\|$ The velocity $\mathcal{V}_{O}$, which corresponds to $\beta_{O} c$ in [18, should not be confused with $v_{0} \equiv \mathcal{V}_{O} / \sqrt{1-e^{2}}$ in $(17-21)$ of [51]. 
and the eccentricity vector $\mathbf{e} \equiv e \mathbf{a}$,

$$
\begin{aligned}
& \left\langle\frac{\mathrm{d} a}{\mathrm{~d} t}\right\rangle_{\mathrm{PN}+\hat{\alpha}_{1}}=0 \\
& \left\langle\frac{\mathrm{d} \mathbf{l}}{\mathrm{d} t}\right\rangle_{\mathrm{PN}+\hat{\alpha}_{1}}=\frac{\hat{\alpha}_{1}}{2 c^{2}} \frac{q-1}{q+1} n_{b} \mathcal{V}_{O} e F_{e}(\mathbf{b} \times \mathbf{w}), \\
& \left\langle\frac{\mathrm{d} \mathbf{e}}{\mathrm{d} t}\right\rangle_{\mathrm{PN}+\hat{\alpha}_{1}}=e \dot{\omega}_{\mathrm{PN}} \mathbf{b}+\frac{\hat{\alpha}_{1}}{2 c^{2}} \frac{q-1}{q+1} n_{b} \mathcal{V}_{O} F_{e}\left(\sqrt{1-e^{2}} w_{a} \mathbf{a}+w_{b} \mathbf{b}-\frac{e^{2} w_{k}}{\sqrt{1-e^{2}}} \mathbf{k}\right),
\end{aligned}
$$

where $q \equiv m_{p} / m_{c}$ is an observable quantity, due to the additional optical information for the two pulsar binaries used in this paper. The three unit vectors $(\mathbf{a}, \mathbf{b}, \mathbf{k})$ form a right-handed triad of a coordinate system with its origin at the center of mass of the binary system, where a points to the position of periastron, $\mathbf{k}$ points along the orbital angular momentum, and $\mathbf{b} \equiv \mathbf{k} \times \mathbf{a}$, as illustrated in figure 1. Furthermore,

$$
F_{e} \equiv \frac{1}{1+\sqrt{1-e^{2}}}
$$

is a function that, for bound orbits $(0 \leq e<1)$, takes a value in the interval $\left[\frac{1}{2}, 1\right)$.

In addition to the contribution from the $\hat{\alpha}_{1}$ and PN terms, we find that for the change caused by the $\hat{\alpha}_{2}$ terms, after averaging over one orbital period,

$$
\begin{aligned}
\left\langle\frac{\mathrm{d} a}{\mathrm{~d} t}\right\rangle_{\hat{\alpha}_{2}} & =0, \\
\left\langle\frac{\mathrm{d} \mathbf{l}}{\mathrm{d} t}\right\rangle_{\hat{\alpha}_{2}} & =\frac{\hat{\alpha}_{2}}{c^{2}} n_{b} F_{e}\left(w_{k} \mathbf{k}+e^{2} F_{e} w_{b} \mathbf{b}\right) \times \mathbf{w}, \\
\left\langle\frac{\mathrm{d} \mathbf{e}}{\mathrm{d} t}\right\rangle_{\hat{\alpha}_{2}} & =\frac{\hat{\alpha}_{2}}{c^{2}} n_{b} F_{e}\left(F_{e} \sqrt{1-e^{2}} w_{a} w_{b} \mathbf{a}-F_{e} \frac{w_{a}^{2}-w_{b}^{2}}{2} \mathbf{b}+w_{b} w_{k} \mathbf{k}\right) e,
\end{aligned}
$$

where $\left(w_{a}, w_{b}, w_{k}\right)$ are the coordinate components of $\mathbf{w}$ in the $(\mathbf{a}, \mathbf{b}, \mathbf{k})$ system.

From (13) and (17) we can see that, as expected, to first order there is no change in the semi-major axis of the orbit from PFEs.

\subsection{Small-eccentricity orbits and PFEs}

The coupled differential equations above simplify considerably for small eccentricities. When $e \ll 1$, one finds $F_{e} \simeq 1 / 2$ and $\mathbf{l} \simeq \mathbf{k}$. To leading order in the (numerically) relevant contributions, (14) and (18) become

$$
\begin{aligned}
\left\langle\frac{\mathrm{d} \mathbf{k}}{\mathrm{d} t}\right\rangle_{\mathrm{PN}+\hat{\alpha}_{1}} & \simeq 0, \\
\left\langle\frac{\mathrm{d} \mathbf{k}}{\mathrm{d} t}\right\rangle_{\hat{\alpha}_{2}} & \simeq \frac{\hat{\alpha}_{2}}{2 c^{2}} n_{b} w_{k} \mathbf{k} \times \mathbf{w},
\end{aligned}
$$

and (15) and (19) simplify to

$$
\begin{aligned}
\left\langle\frac{\mathrm{d} \mathbf{e}}{\mathrm{d} t}\right\rangle_{\mathrm{PN}+\hat{\alpha}_{1}} & \simeq e \dot{\omega}_{\mathrm{PN}} \mathbf{b}+\frac{\hat{\alpha}_{1}}{4 c^{2}} \frac{q-1}{q+1} n_{b} \mathcal{V}_{O} \mathbf{w}_{\perp}, \\
\left\langle\frac{\mathrm{d} \mathbf{e}}{\mathrm{d} t}\right\rangle_{\hat{\alpha}_{2}} & \simeq 0,
\end{aligned}
$$


where $w \equiv|\mathbf{w}|$ and $\mathbf{w}_{\perp} \equiv w_{a} \mathbf{a}+w_{b} \mathbf{b}$ is the projection of $\mathbf{w}$ into the orbital plane. Above four equations have been derived, under the consideration that $e \lesssim 10^{-6}$, $w^{2} / c^{2} \sim \mathcal{V}_{O} w / c^{2} \sim \mathcal{V}_{O}^{2} / c^{2} \sim 10^{-6}$, for the NS-WD systems which are to be used in our calculations, i.e., PSRs J1012+5307 and J1738+0333 (see table 1).

From (20) and (22), Damour and Esposito-Farèse [12 worked out the eccentricity vector evolution under the influence of the PN and $\hat{\alpha}_{1}$ terms, which turns out to be a superposition of a $\mathrm{PN}$-induced precessing eccentricity $\mathbf{e}_{R}(t)$, and a constant "forced eccentricity" $\mathbf{e}_{F}$, introduced by $\hat{\alpha}_{1}$.

In terms of a geometrical interpretation of the time evolution of the orbital eccentricity, the physical consequence of $\hat{\alpha}_{1}$ was extensively studied [12, 6, 50]. The physical consequence of $\hat{\alpha}_{2}$ for small-eccentricity binary systems is readily derived from (21), which shows that a non-zero $\hat{\alpha}_{2}$ causes a precession of the orbital angular momentum around the fixed direction $\mathbf{w}$ with an angular frequency,

$$
\Omega_{\hat{\alpha}_{2}}^{\mathrm{prec}}=-\frac{\hat{\alpha}_{2}}{2} n_{b}\left(\frac{w}{c}\right)^{2} \cos \psi,
$$

where $\psi$ is the angle between $\mathbf{k}$ and $\mathbf{w}$ (see figure $\mathbf{1}$ for an illustration of the orbital geometry and the orbital angular momentum precession). To leading order, this precession is purely determined by $\hat{\alpha}_{2}$ (see (20) ).

The precession (24) induces a secular change of the projected semi-major axis of the pulsar orbit. The rate of change is given by

$$
\left(\frac{\dot{x}}{x}\right)_{\hat{\alpha}_{2}}=-\frac{\hat{\alpha}_{2}}{4} n_{b}\left(\frac{w}{c}\right)^{2} \cot i \sin 2 \psi \cos \vartheta,
$$

where $\vartheta$ is the angle between $\mathbf{w}_{\perp}$ and the direction of ascending node. In section 3 , we will apply (25) to constrain $\hat{\alpha}_{2}$ from two relativistic small-eccentricity NS-WD binaries, namely, PSRs J1012+5307 and J1738+0333.

\subsection{The preferred frame}

As the most natural preferred frame for our following calculations, we choose the frame determined by the isotropic CMB, like this is generally done in the literature on preferred-frame tests. To use other frames, the generalization is straightforward. As an example, in section 5 we also present limits on $\hat{\alpha}_{1}$ and $\hat{\alpha}_{2}$ for which the Galaxy or the local Galactic rotation is assumed to determine the preferred frame.

From the five-year Wilkinson Microwave Anisotropy Probe (WMAP) operations, a CMB dipole measurement of $3.355 \pm 0.008 \mathrm{mK}$ was obtained, which implies a peculiar velocity of the Solar system barycenter (SSB) with respect to the CMB frame of $\left|\mathbf{v}_{\mathrm{SSB}-\mathrm{CMB}}\right|=369.0 \pm 0.9 \mathrm{~km} \mathrm{~s}^{-1}$, in the direction of Galactic longitude and latitude $(l, b)=\left(263.99^{\circ} \pm 0.14^{\circ}, 48.26^{\circ} \pm 0.03^{\circ}\right)$ [21]. Results from the seven-year WMAP observations are unchanged [24]. The binary velocity with respect to the preferred frame is $\mathbf{w}=\mathbf{v}_{\text {PSR-SSB }}+\mathbf{v}_{\text {SSB-CMB }}$, where $\mathbf{v}_{\text {PSR-SSB }}$ is the 3-dimensional (3D) motion of the pulsar binary system, with respect to the SSB. For PSRs J1012+5307 and J1738+0333, $\mathbf{v}_{\mathrm{SSB}-\mathrm{CMB}}$ can be derived from a combination of the distance and proper 
motion measurements from radio timing, and the radial velocity obtained from spectral observations of the WD.

\section{New constraints on $\hat{\alpha}_{2}$}

To constrain $\hat{\alpha}_{2}$ from binary pulsar observations, we start from (25), where the orbital frequency, $n_{b}=2 \pi / P_{b}$, and the projected semi-major axis, $x$, are observable Keplerian parameters, while the time derivative of $x, \dot{x}$, belongs to the set of phenomenological post-Keplerian parameters [10, 18]. They are obtained with high precision from radio timing observations. In (25), we also need the inclination of the binary orbit with respect to the line of sight $i$. For the binaries of this paper, $i$ can be determined (modulo the ambiguity of $i \rightarrow 180^{\circ}-i$ ) from the mass function, leading to

$$
\sin i=\frac{c x n_{b}}{\mathcal{V}_{O}}(q+1) \text {. }
$$

The companion mass $m_{c}$ is inferred from spectroscopic and photometric studies of the WD companion using well tested atmospheric model for such WDs [8, 1]. The pulsar mass $m_{p}$ is determined from the mass ratio of the pulsar and its companion, $q \equiv m_{p} / m_{c}$, which is inferred from the radial velocity and the orbital parameters of the binary system [8, 1]. Unfortunately, the information is not sufficient to calculate $\sin i$, since

$\mathcal{V}_{O}$ contains, besides the known total mass $M$, the effective gravitational constant $\hat{G}$ which is a priori unknown if one does not specify a given gravity theory. In principle, strong-field modification could lead to a significant deviation of $\hat{G}$ from the GR value, $G$. Such modifications, on the other hand, are expected to be accompanied by a significant amount of dipolar gravitational radiation (as an example, see [12] for the case of tensorscalar theories of gravity), which is neither the case in PSR J1012+5307 [34] nor in PSR J1738+0333 [20]. Consequently, for the required precision in $i$, we can safely assume $\hat{G} \simeq G$ in (26).

In order to fully determine the orientation of the binary with respect to $\mathbf{w}(\psi$ and $\vartheta$ in (25)), one also needs the longitude of the ascending node, $\Omega$, an angle which (in most cases) is not measurable from pulsar timing experiments. Consequently, in our $\hat{\alpha}_{2}$ tests we will treat $\Omega$ as a random variable uniformly distributed between $0^{\circ}$ and $360^{\circ}$. This, however, will require probabilistic arguments in order to exclude those (small) ranges of $\Omega$ where $\hat{\alpha}_{2}$ would practically be unconstrained.

\subsection{PSR J1012+5307}

PSR J1012+5307 is a small-eccentricity NS-WD binary system, with an orbital period of $\sim 14.5 \mathrm{~h}$. The pulsar was discovered in 1993 with the $76-\mathrm{m}$ Lovell radio telescope at Jodrell Bank [41, and optical observations revealed its companion being a helium WD [36]. Callanan et al [8] measured a systemic radial velocity of $44 \pm 8 \mathrm{~km} \mathrm{~s}^{-1}$ relative to the $\mathrm{SSB}$, the mass ratio $q=10.5 \pm 0.5$, and the companion mass $m_{c}=0.16 \pm 0.02 \mathrm{M}_{\odot}$.

Lange et al [33] used 4-year Effelsberg 100-m radio telescope timing data and 7-year 76-m Lovell telescope timing data to derive a set of pulsar timing parameters, 

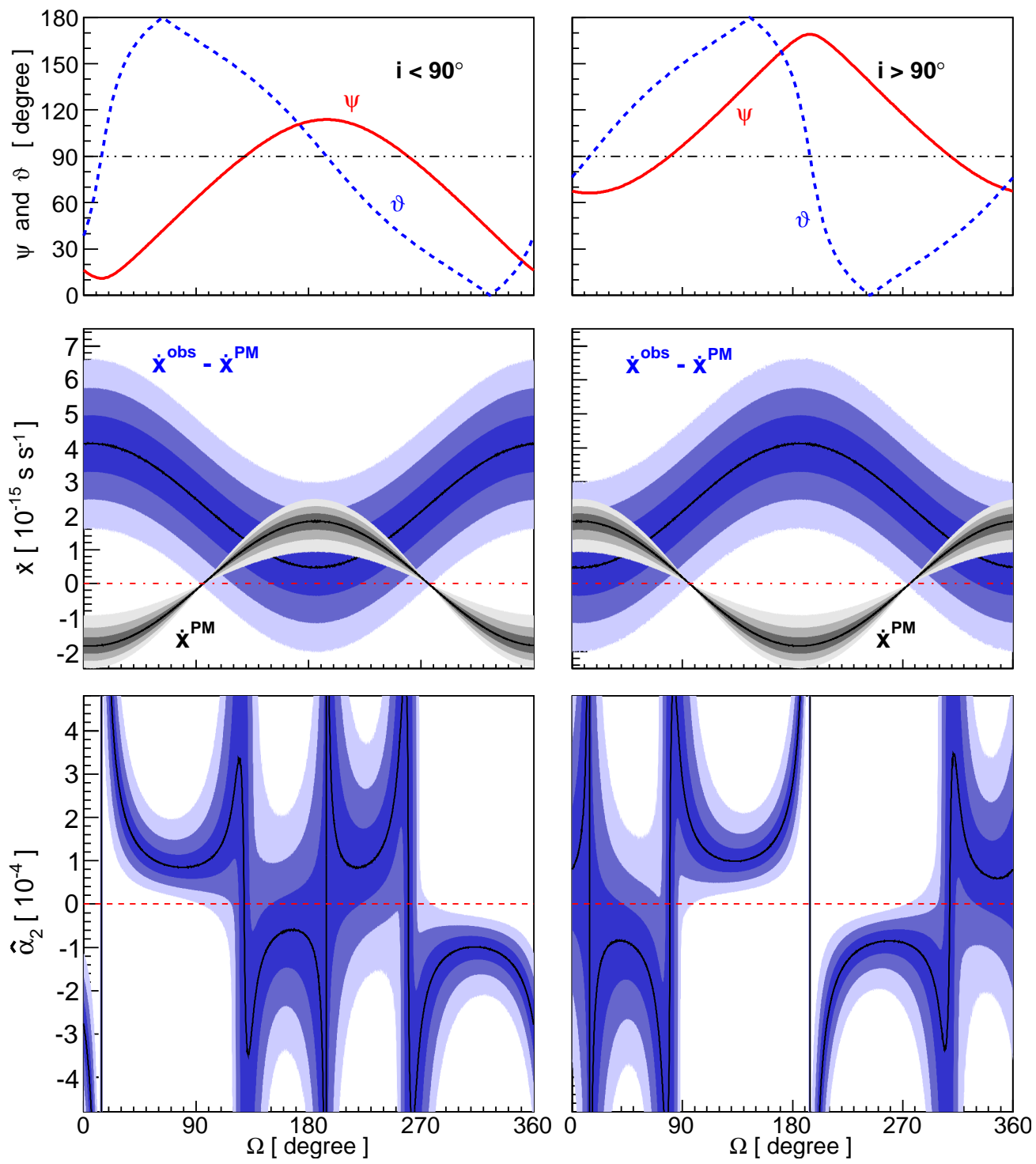

Figure 2. Upper: Illustration of two important angles of the PSR J1012+5307 binary in our calculation, as a function of the unobservable longitude of the ascending node $\Omega$ : $\psi$, the angle between $\mathbf{w}$ and the orbital angular momentum, and $\vartheta$, the angle between $\mathbf{w}_{\perp}$ and the direction of ascending node. Middle: Proper motion contribution to $\dot{x}$, namely $\dot{x}^{\mathrm{PM}}$ (gray), and the residual $\dot{x}$, namely $\dot{x}^{\text {obs }}-\dot{x}^{\mathrm{PM}}$ (blue). Lower: Derived $\hat{\alpha}_{2}$ from the residual $\dot{x}$. Different contours correspond to $68 \%, 95 \%$, and $99.7 \%$ confidence levels. The left column is for the $i<90^{\circ}$ branch, and the right column is for the $i>90^{\circ}$ branch. 
by utilizing the low eccentricity binary timing model ELL1. These timing results were used to put stringent limits on the emission of dipolar gravitational radiation by this NS-WD system. Most recently, Lazaridis et al 34] updated the timing parameters by using 15 years of observations from the European Pulsar Timing Array (EPTA) network, consisting of the four radio-telescopes Effelsberg (Germany), Jodrell Bank (UK), Westerbork (the Netherlands) and Nançay (France).

The short orbital period and the measurement of its spatial systemic velocity make the PSR J1012+5307 system particularly interesting for studies of PFEs and tests of the corresponding parameters. To utilize (25), the full information of $\mathbf{w}$ and the orientation of the orbital plane is needed. To get $\mathbf{w}$, we calculate the binary velocity with respect to SSB from the transverse velocity obtained from radio timing and the radial velocity measurement via spectroscopy of the WD companion. With these measurements at hand one can compute $\mathbf{w}=\mathbf{v}_{\mathrm{PSR}-\mathrm{SSB}}+\mathbf{v}_{\mathrm{SSB}-\mathrm{CMB}}$. The inclination of the orbital plane, $i$, is calculated from (26), with a sign ambiguity between $i<90^{\circ}$ and $i>90^{\circ}$. Hence we have two branches of solution. Moreover, pulsar timing experiments generally give no information on the longitude of the ascending node $\Omega$. We sample it in the range $\left[0,360^{\circ}\right)$. Figure 2 illustrates $\psi$ (angle between $\mathbf{w}$ and the orbital angular momentum) and $\vartheta$ (angle between the ascending node and $\mathbf{w}_{\perp}$ ), as a function of $\Omega$. In the figures of $\psi$ and $\vartheta$, measurement uncertainties of $m_{c}, q$, and the proper motion are not included. However, our simulations to constrain $\hat{\alpha}_{2}$, which are to be discussed below, take full account of all measurement uncertainties.

To look into the change of $x$ induced by the $\hat{\alpha}_{2}$ term, we should separate other potential effects from the measured $\dot{x}$. A change of $x$ can come from various astrophysical and gravitational effects [18, 35]. All effects that cause a change to the semi-major axis of the system, like gravitational wave damping and component mass loss, can be constrained observationally via the observed $\dot{P}_{b}^{\text {obs }}$ (see table 1). In fact, we can re-write $(8.76)$ in [35] to

$$
\left(\frac{\dot{x}}{x}\right)^{\mathrm{obs}}=\frac{2}{3}\left(\frac{\dot{P}_{b}}{P_{b}}\right)^{\mathrm{obs}}-\frac{\dot{D}}{3 D}+\left(\frac{\dot{x}}{x}\right)^{\mathrm{PM}}+\frac{\mathrm{d} \varepsilon_{A}}{\mathrm{~d} t}+\left(\frac{\dot{x}}{x}\right)^{\mathrm{SO}}+\left(\frac{\dot{x}}{x}\right)^{\text {planet }} .
$$

The remaining terms are due to a change in the Doppler factor $D$, the proper motion of the binary system, a change in the aberration due to a change in the pulsar-spin orientation, a change in the orbital inclination due to spin-orbit coupling effects, and finally a mass distribution in the vicinity of the system. Before calculating $\hat{\alpha}_{2}$ from (25), these influences on $\dot{x}$ should be subtracted. We will discuss them term by term in the following.

Using the measured quantities of PSR J1012+5307 in table 1, the first term on the right-hand side of (27) can be estimated to be $\dot{x}^{\dot{P}_{b}} \sim 4 \times 10^{-19} \mathrm{~s} \mathrm{~s}^{-1}$, which is four orders of magnitude smaller than the relevant scale.

The second term, $-\dot{D} / 3 D$, includes contributions from the Galactic acceleration of the binary system, and the Shklovskii effect that is induced by the transverse proper 
motion [46]. One finds [16]

$$
-\frac{\dot{D}}{D}=\frac{1}{c} \mathbf{K}_{0} \cdot\left(\mathbf{g}_{\mathrm{PSR}}-\mathbf{g}_{\mathrm{SSB}}\right)+\frac{v_{T}^{2}}{c d}
$$

where $\mathbf{K}_{0}$ is the unit vector pointing from the SSB to the binary pulsar, as defined before, $d$ is the pulsar distance from the SSB, $v_{T}=d \sqrt{\mu_{\alpha}^{2}+\mu_{\delta}^{2}}$ is the transverse velocity of the system with respect to the $\mathrm{SSB}, \mathbf{g}_{\mathrm{PSR}}$ and $\mathbf{g}_{\mathrm{SSB}}$ are the Galactic accelerations of the binary and the SSB, respectively. The contributions from the Galactic acceleration and the Shklovskii effect to $\dot{x}$ are of order $2 \times 10^{-20} \mathrm{~s} \mathrm{~s}^{-1}$ and $3 \times 10^{-19} \mathrm{~s} \mathrm{~s}^{-1}$, respectively. Hence, both contributions are negligible [34.

The third term of (27) is a variation of $x$ caused by a change of the orbital inclination $i$, due to the proper motion of the binary system [2, 29],

$$
\left(\frac{\dot{x}}{x}\right)^{\mathrm{PM}}=\left(-\mu_{\alpha} \sin \Omega+\mu_{\delta} \cos \Omega\right) \cot i .
$$

This contribution is not negligible. The contribution from the proper motion effect is depicted in the middle panels of figure 2 for $i<90^{\circ}$ (left) and $i>90^{\circ}$ (right), respectively, as a function of the unknown longitude of the ascending node $\Omega$. In [34] the $\dot{x}$ measurement was used to constrain $\Omega$, by assuming that the measured $\dot{x}$ is solely caused by the proper motion effect (29). They got constraints on $\Omega$, by requiring $\dot{x}^{\text {obs }}=\dot{x}^{\mathrm{PM}}$ (see (11-14) in [34]). In our test we have to keep full ignorance of $\Omega$, and cannot assume $\dot{x}^{\text {obs }}=\dot{x}^{\mathrm{PM}}$. The residual $\dot{x}$, after subtracting of the contribution from binary proper motion, is also plotted in the same figure. The intersections of $\dot{x}^{\text {obs }}-\dot{x}^{\mathrm{PM}}$ and the horizontal null lines in the figure correspond to the limit on $\Omega$ obtained in [34].

The fourth term in (27) is due to the varying aberration caused by geodetic precession of the pulsar spin axis [18]. For a nearly circular orbit one finds

$$
\frac{\mathrm{d} \varepsilon_{A}}{\mathrm{~d} t} \simeq-\frac{P}{P_{b}} \frac{\cot \lambda \sin 2 \eta+\cot i \cos \eta}{\sin \lambda} \Omega_{\mathrm{geod}}
$$

where $P$ is the pulsar spin period, $\lambda$ and $\eta$ are positional angles of the pulsar spin vector (see figure 1 in [18] for details). In GR, the geodetic precession rate for a nearly circular orbit is given by [4]

$$
\Omega_{\text {geod }} \simeq \frac{3+4 q}{2(1+q)^{2}}\left(\frac{\mathcal{V}_{O}^{(\mathrm{GR})}}{c}\right)^{2} n_{b} .
$$

Consequently, from the timing parameters in table 1 one finds that the $\mathrm{d} \varepsilon_{A} / \mathrm{d} t$ term produces a change of $x$ of order $10^{-18} \mathrm{~s} \mathrm{~s}^{-1}$ for typical spin orientations. Hence, it is negligible in our case, unless there is a deviation from GR by at least a factor of 100, which we consider as highly unlikely, as such a large deviation of gravity in this system is clearly not seen in the gravitational wave emission [34]. Moreover, PSR J1012+5307 is a highly recycled pulsar, and therefore its spin axis is expected to be nearly aligned with the orbital angular momentum (i.e. $\eta \simeq-90^{\circ}$ and $\lambda \simeq i$ ), which greatly suppresses this effect anyway. 
The classical spin-orbital coupling, due to the quadrupole moment of the companion star, can change the inclination of the orbital plane, which induces the fifth term of (27). But this effect is only important for main-sequence star companions [26, 49] or rapidly rotating WD companions [27], and can be neglected here.

The last term of (27) is only valid if there is a nearby third companion, that perturbs the orbit significantly, like in the PSR B1620-26 system [2]. This is not the case for PSR J1012+5307 [34], as this would be well seen in the presence of higher oder derivatives of the rotational frequency of the pulsar [25].

In summary, only the proper motion term (29) is important in our studies here. After accounting for this effect on $\dot{x}^{\text {obs }}$ for every given $\Omega$, we calculate the contour plots of $\hat{\alpha}_{2}$, and present them in the lower panels of figure 2, for $i<90^{\circ}$ (left) and $i>90^{\circ}$ (right), respectively. In the calculation, $10^{5}$ Monte Carlo simulations are implemented to account for the measurement uncertainties of $\mu_{\alpha}, \mu_{\delta}, d, v_{r}, q, m_{c}$, and $\dot{x}$. As we can see, $\hat{\alpha}_{2}$ can be constrained to the order of $10^{-4}$ for most $\Omega$ realization. The $\Omega$ values in the figure where $\hat{\alpha}_{2}$ is virtually unconstrained correspond to the configurations when the angle between $\mathbf{w}$ and the orbital angular momentum $\psi \simeq 90^{\circ}$, or the angle between the projected $\mathbf{w}$ onto the orbital plane $\mathbf{w}_{\perp}$ and the direction of the ascending node $\vartheta \simeq 90^{\circ}$ (see horizontal lines in the upper panels of figure 2 for corresponding angles and compare them with the divergencies in the lower panels). The reason for the divergencies is easy to see from (25), where the right hand side vanishes when $\psi=90^{\circ}$, or $\vartheta=90^{\circ}$, independent of $\hat{\alpha}_{2}$. In this situation $\hat{\alpha}_{2}$ cannot be constrained.

\section{2. $P S R J 1738+0333$}

PSR J1738+0333 is a small-eccentricity NS-WD binary system, with an orbital period of $\sim 8.5 \mathrm{~h}$, which, together with other well measured physical quantities, makes it a superb astrophysical laboratory to test gravitational theories [20]. The pulsar was discovered in 2001 in the Parkes high Galactic latitude survey [23], and later regularly timed with the 305-m Arecibo telescope [20]. It is one of the four millisecond pulsars known to be orbited by a WD companion bright enough for detailed spectroscopy [1], among which, PSR J1738+0333 is the most relativistic. Detailed optical studies of the WD companion and radio timing studies of the pulsar are presented in [1] and [20], respectively. Thanks to their studies, accurate binary parameters and spatial motion (transverse and radial) are available for the PSR J1738+0333 binary system. Therefore, it also presents a good laboratory to study PFEs.

The strategy to constrain $\hat{\alpha}_{2}$ is the same as in the case of PSR J1012+5307. First, we get $\mathbf{w}$ from $\mathbf{v}_{\mathrm{PSR}-\mathrm{SSB}}$ and $\mathbf{v}_{\mathrm{SSB}-\mathrm{CMB}}$, and then the configuration of the system with respect to the CMB frame can be obtained, as a function of $\Omega$, with a sign ambiguity of $i$. Two important angles, $\psi$ and $\vartheta$, are depicted in the upper panels of figure 3 , for $i<90^{\circ}$ (left) and $i>90^{\circ}$ (right), respectively.

Along the line of arguments for PSR 1012+5307 one finds, using the results of [20] (see table 1 for binary parameters), that also for PSR J1738+0333 the only relevant 

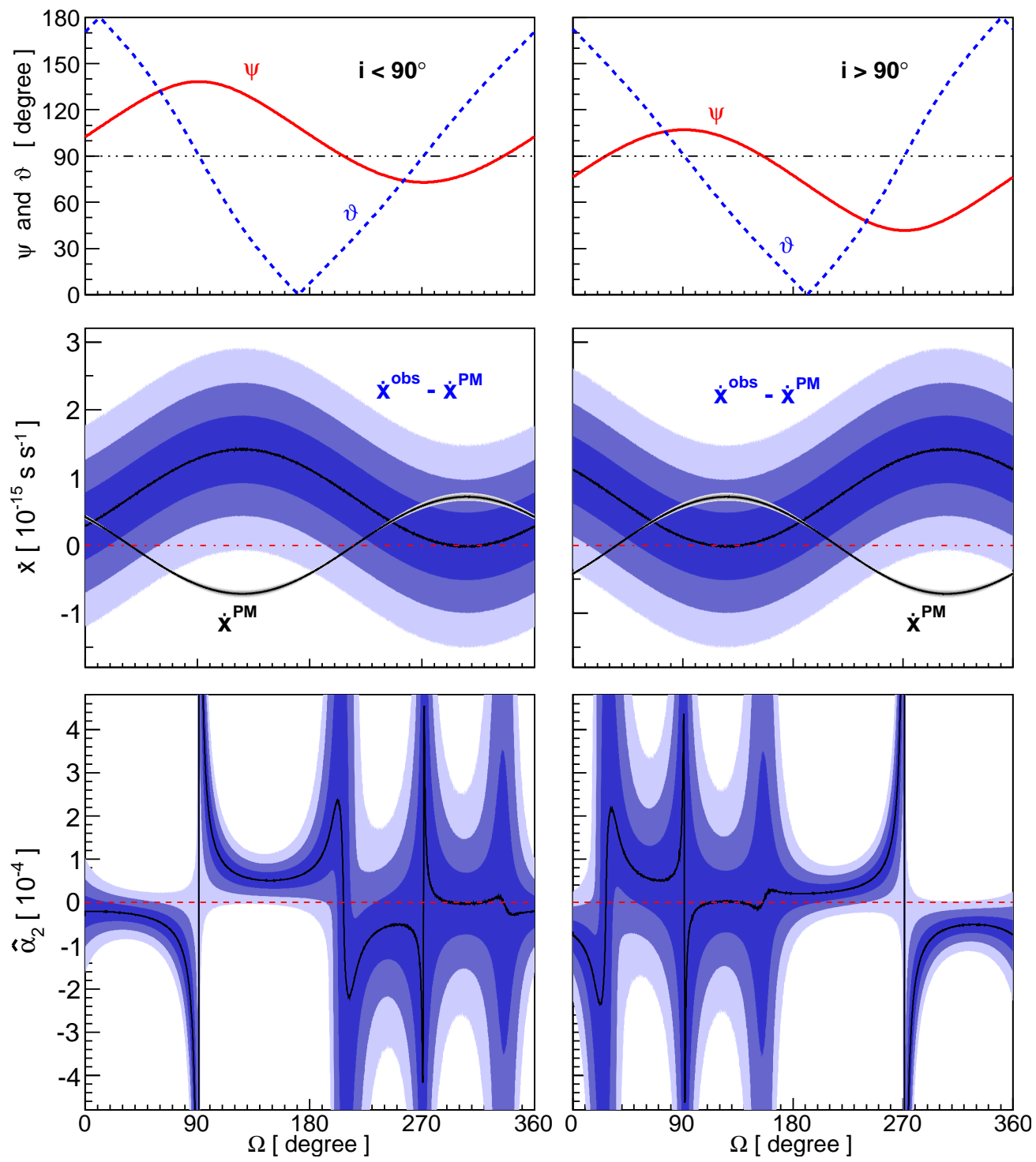

Figure 3. Same as figure 2, for PSR J1738+0333.

term in (27) is the contribution by the proper motion of the system, $\dot{x}^{\mathrm{PM}}$. Because of the more precise measurements of the PSR J1738+0333 parameters, the uncertainty of $\dot{x}^{\mathrm{PM}}$ is smaller correspondingly, as illustrated in the middle panels of figure 3 for $i<90^{\circ}$ (left) and $i>90^{\circ}$ (right), respectively. The corresponding residual values for $\dot{x}$, after subtracting $\dot{x}^{\mathrm{PM}}$, are also depicted.

If we adopt the assumption that GR is the correct theory of gravity for the PSR J1738+0333 system, then we can get constraints on the longitude of the ascending 


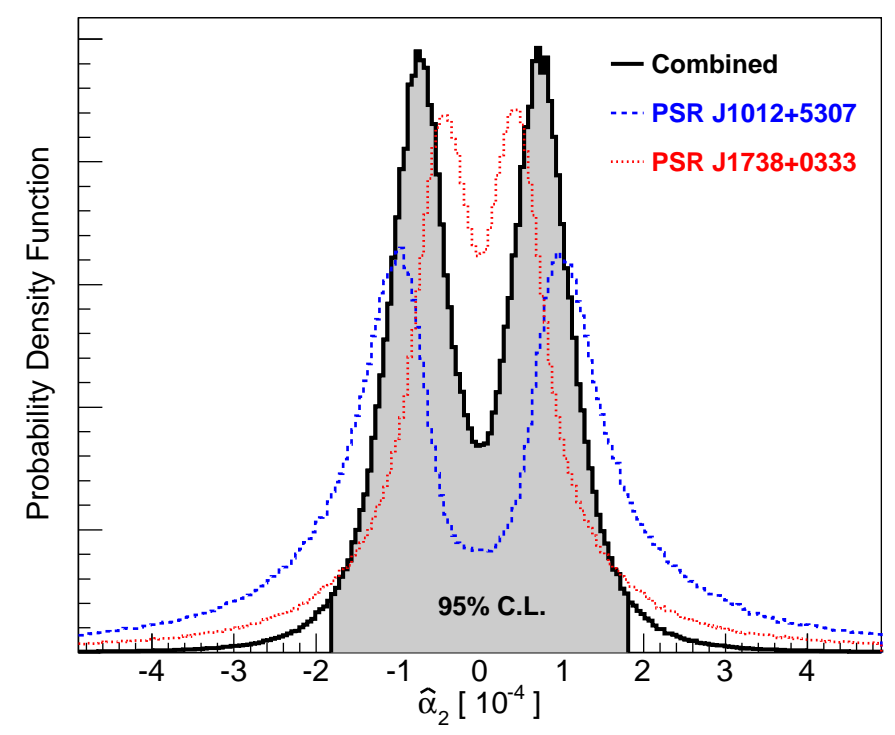

Figure 4. Probability distributions of $\hat{\alpha}_{2}$ from PSR J1012+5307 (blue dashed histogram), PSR J1738+0333 (red dotted histogram), and their combination (black solid histogram). At $95 \%$ confidence level, $\left|\hat{\alpha}_{2}\right|$ is constrained to be less than $1.8 \times 10^{-4}$ from the combined probability distribution.

node $\Omega$. At a $95 \%$ confidence level one finds

$$
\begin{array}{ll}
\Omega \in\left(0^{\circ}, 60^{\circ}\right) \text { or }\left(190^{\circ}, 360^{\circ}\right) & \text { when } i<90^{\circ}, \\
\Omega \in\left(10^{\circ}, 240^{\circ}\right) & \text { when } i>90^{\circ} .
\end{array}
$$

We will partly use these results in the next section, where we constrain $\hat{\alpha}_{1}$. Naturally, to constrain $\hat{\alpha}_{2}$ we cannot use (32) as it is based on GR, i.e. $\hat{\alpha}_{2} \equiv 0$. The same is true for PSR 1012+5307, where the constraint of $\Omega$ can be found in (11-14) of [34].

In the calculation, for every $\Omega 10^{5}$ Monte Carlo simulations are implemented to account for the measurement uncertainties of $\mu_{\alpha}, \mu_{\delta}, d, v_{r}, q, m_{c}$, and $\dot{x}$. The results are plotted in the lower panels of figure 3 for $i<90^{\circ}$ (left) and $i>90^{\circ}$ (right), respectively. Similarly, divergencies are caused by the unfavorable configurations with $\psi \simeq 90^{\circ}$ or $\vartheta \simeq 90^{\circ}$ (see upper panels for reference), where $\hat{\alpha}_{2}$ can hardly be constrained. For most $\Omega, \hat{\alpha}_{2}$ is constrained to be of order $\sim 5 \times 10^{-5}$, about two times better than that of PSR J1012+5307.

\subsection{Probability distribution of $\hat{\alpha}_{2}$}

We cannot directly constrain $\hat{\alpha}_{2}$ from (25), and have to set up Monte Carlo simulations to account for our ignorance in $\Omega$ and ambiguity between $i$ and $180^{\circ}-i$. In the simulation, we assume that $\Omega$ is uniformly distributed between $0^{\circ}$ and $360^{\circ}$, and an equal probability to have $i<90^{\circ}$ or $i>90^{\circ}$. The measurement uncertainties of the spatial binary motions, the time derivative of the projected semi-major axis $\dot{x}$, the binary mass ratio $q$, the companion mass $m_{c}$, and also the distance from parallax measurement, are properly 
accounted for, assuming a Gaussian error distribution. One million simulations for each of the two pulsars have been conducted. In every realization, we subtract the secular effects on $\dot{x}$ from proper motions, and calculate $\hat{\alpha}_{2}$ according to (25).

The simulated results are summarized as probability distributions of $\hat{\alpha}_{2}$ for PSRs J1012+5307 and J1738+0333, illustrated in figure 4using a blue dashed histogram and a red dotted histogram, respectively. The long tails of the probability distributions from these two binaries are due to the undesirable configurations when $\psi \simeq 90^{\circ}$ or $\vartheta \simeq 90^{\circ}$, which cause the divergencies in the lower panels of figures 2 and 3 , From these probability distributions we find at $95 \%$ confidence level,

$$
\begin{array}{ll}
\left|\hat{\alpha}_{2}\right|<3.6 \times 10^{-4} & \text { for PSR J1012+5307, } \\
\left|\hat{\alpha}_{2}\right|<2.9 \times 10^{-4} & \text { for PSR J1738+0333. }
\end{array}
$$

In figure 4, we also show the probability distribution of $\hat{\alpha}_{2}$ from the combination of these two pulsar binaries (black solid histogram), assuming that their measurements are independent, and that $\hat{\alpha}_{2}$ has only a weak functional dependence on the NS mass in the range of $1.3-2.0 \mathrm{M}_{\odot}$. The combined probability distribution demonstrates a much shorter and suppressed tail, which means it is very unlikely that both systems are in the unfavorable configurations. From the combined probability distribution, we obtain a constraint of

$$
\left|\hat{\alpha}_{2}\right|<1.8 \times 10^{-4} \quad(95 \% \text { C.L. }) \text {. }
$$

It is by three orders of magnitude better than the result of [51], i.e. (44), although one has to keep in mind that the double pulsar tests the gravitational interaction of two strongly self-gravitating objects. This limit is still by three orders of magnitude weaker than the Solar system limit (2), but accounts for possible strong-field deviations in NSs. Compared with the Solar system limit from LLR [39, 35) is still 3.6 times weaker. Being related to a secular effect, the limit on $\hat{\alpha}_{2}$ will improve fast with observing time

$T_{\text {obs }}$, namely proportional to $T_{\text {obs }}^{-3 / 2}$. A disadvantage of this test, as compared for instance to the LLR experiment, is its dependence on probabilistic considerations with respect to the unknown angle $\Omega$.

\section{A robust method to constrain $\hat{\alpha}_{1}$}

As mentioned in section 2, Damour and Esposito-Farèse used a novel geometrical way to constrain $\hat{\alpha}_{1}$ with small-eccentricity binary pulsars [12]. In their paper they showed that the observed eccentricity vector, $\mathbf{e}(t)$, is a vectorial superposition of a "rotating eccentricity" $\mathbf{e}_{R}(t)$ (with constant length), and a "forced eccentricity" $\mathbf{e}_{F}$,

$$
\mathbf{e}(t)=\mathbf{e}_{F}+\mathbf{e}_{R}(t),
$$

where $\mathbf{e}_{R}(t)$ is a vector of (unknown) constant magnitude which rotates in the orbital plane with angular velocity $\dot{\omega}_{\mathrm{PN}}$, and $\mathbf{e}_{F}$ is a fixed vector,

$$
\mathbf{e}_{F}=\frac{\hat{\alpha}_{1}}{4 c^{2}} \frac{q-1}{q+1} \frac{n_{b}}{\dot{\omega}_{\mathrm{PN}}} \mathcal{V}_{O} \mathbf{k} \times \mathbf{w} .
$$




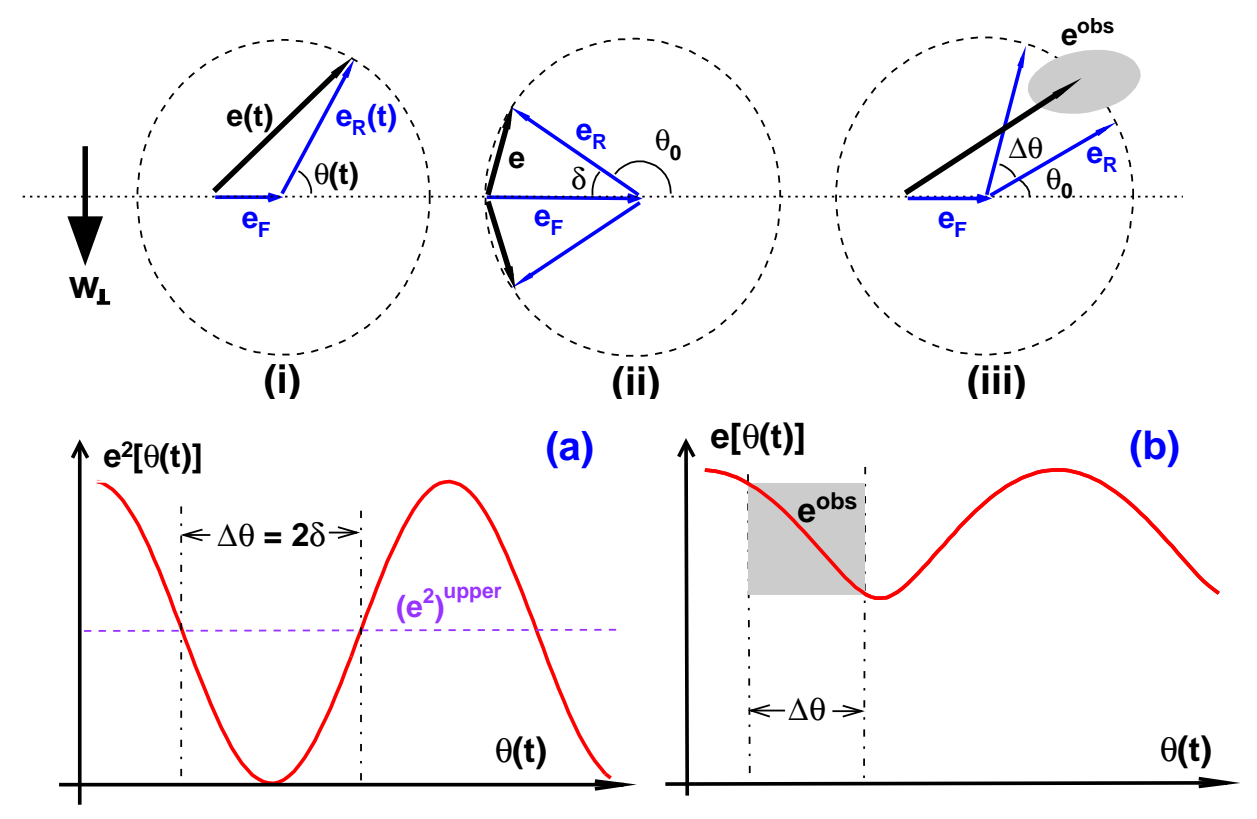

Figure 5. Illustration of our robust method to constrain $\hat{\alpha}_{1}$. Upper: (i) observational eccentricity $\mathbf{e}(t)$, is a vectorial superposition of a "rotating eccentricity" $\mathbf{e}_{R}(t)$ and a "forced eccentricity" $\mathbf{e}_{F}$ [12] (ii) the worst starting configuration, where $\mathbf{e}_{R}(t)$ rotates $\Delta \theta=2 \delta$ during the observational time, and in the midtime, $\mathbf{e}_{R}(t)$ and $\mathbf{e}_{F}$ cancel out completely; this applies to binaries where no explicit eccentricity is detected, like in PSR J1012+5307, and it constrains $\hat{\alpha}_{1}$ most conservatively; (iii) during the observational time, $\mathbf{e}_{R}(t)$ rotates out an angle $\Delta \theta$, and the time-averaged $\mathbf{e}$ and its variance should be consistent with measurements (see text for two criteria); this applies to binaries where an explicit eccentricity vector is detected, like in PSR J1738+0333. Lower: (a) the sinusoidal evolution of $e^{2}(t)$ as a function of $\theta(t)=\theta_{0}+\dot{\omega}_{\mathrm{PN}} t$; the indicated $\Delta \theta$ corresponds to the one of the upper panel (ii); (b) the (not sinusoidal) time evolution of $e(t)$ as a function of $\theta(t)$, and the indicated $\Delta \theta$ corresponds to the one of the upper panel (iii).

A graphical illustration of this dynamics is given in the upper panel (i) of figure 5 ,

In analogy with the equivalence principle violation test in [11, a probabilistic reasoning is used in [12] to constrain $\hat{\alpha}_{1}$. Because of two unknown angles, i.e., the timedependent angle $\theta(t)$ between $\mathbf{e}_{F}$ and $\mathbf{e}_{R}(t)$, and the unknown angle of the longitude of the ascending node, $\Omega$, the probabilistic method has to assume a uniform distribution of $\theta(t)$ and $\Omega$ between $0^{\circ}$ and $360^{\circ}$. To make the "random $\theta(t)$ " argument plausible, the binaries used in the test should be old enough to let $\mathbf{e}_{R}(t)$ have rotated several cycles [12, 11]. Above two restrictions can be dropped in our robust method below.

In order to address a potential selection effect in the test of [12], when picking the system with the best figure of merit from a whole population of binary pulsars, an $\hat{\alpha}_{1}$ test has been developed in [50] that extends the method of [12] to the full related population, including those systems that have a low figure of merit. However, from the viewpoint of alternative gravity theories, the $\hat{\alpha}_{1}$ parameter might depend on the specific 
masses of the binary components used in the analysis. Such a dependence is well known for the generalized PPN parameters $\hat{\gamma}$ and $\hat{\beta}$ [52]. But in an analysis that combines different binary pulsar systems, one is forced to a priori assume a weak dependence of $\hat{\alpha}_{1}$ on the NS mass. Our new robust method can also naturally overcome the issues concerning the selection effect and the mass dependence, as it is based on a direct test of secular changes caused by $\hat{\alpha}_{1}$ in individual systems.

The basic idea behind our robust method is the expected change in the eccentricity vector during the observational span $T_{\text {obs }}$, in case of a non-vanishing $\hat{\alpha}_{1}$. Because of the considerable periastron advance (see table 1 for GR values), and the fact that, by now, the timing observations span more than 10 years for PSRs J1012+5307 and J1738+0333, the "rotating eccentricity" $\mathbf{e}_{R}(t)$ has already swept out a sizable angle $\Delta \theta\left(\geq 10^{\circ}\right.$ for both PSRs J1012+5307 and J1738+0333). In the presence of a large $\mathbf{e}_{F}$, this would induce an observable change of the eccentricity vector (see figure 5). We can use the tight constraints on the very small eccentricities of these two systems to limit such a variation of the eccentricity vector, and use this to directly constrain $\hat{\alpha}_{1}$ with remarkable precision.

We will distinguish between two cases, depending on whether an upper bound or a positive measurement of the eccentricity vector is made from observations. If the eccentricity is not measured (like for PSR J1012+5307), we use the measured (small) limit on the eccentricity to constrain $e_{F}$, and consequently $\hat{\alpha}_{1}$. If a positive detection of the eccentricity vector is made (like for PSR J1738+0333), we include its directional information along with the smallness of its variation to constrain $\hat{\alpha}_{1}$.

\subsection{PSR J1012+530\%, a short orbital period system with an unmeasured eccentricity}

For PSR J1012+5307, at present there is no measurement of an orbital eccentricity, but a tight upper limit of the order of $10^{-7}$ (see table 1). This low limit, in combination with the fact that the periastron should have precessed by about $10^{\circ}$ over the observing time span $T_{\text {obs }}$, allows to put constraints on an $\hat{\alpha}_{1}$-related polarization of the orbit. In the following we will outline the method.

From (36), we arrive at,

$$
e^{2}=e_{F}^{2}+e_{R}^{2}+2 e_{F} e_{R} \cos \theta .
$$

Since $e_{R}$ and $e_{F}$ are both constant, the observational $e^{2}[\theta(t)]$ changes as a sinusoidal function of $\theta(t)$, as shown in the lower panel (a) of figure 5. As $\theta(t)=\theta_{0}+\dot{\omega}_{\mathrm{PN}} t$ is a linear function of time $t, e^{2}(t)$ is a sinusoidal function of $t$ as well. We also show a typical temporal evolution of $e[\theta(t)]$ in the lower panel (b) of figure [5, which is not sinusoidal.

For relativistic small-eccentricity binary systems, especially for those that are being observed for more then a decade, the vector $\mathbf{e}_{R}(t)$ has already swept out a non-negligible angle, which corresponds in the lower panels of figure 5 to a span $\Delta \theta$ in the horizontal axis. This factor would induce an eccentricity variance because of the vectorial addition in (36) that scales with $\hat{\alpha}_{1}$. If then over a long time span an orbital eccentricity remains undetected to a small value, like for PSR J1012+5307, one can directly constrain $\left|\hat{\alpha}_{1}\right|$ 
to some upper limit $\left|\hat{\alpha}_{1}\right|^{\text {upper }}$, without any probabilistic assumptions about $\theta$. The reasoning is as follows. If $e_{F} \gg e_{R}$, then $\mathbf{e} \simeq \mathbf{e}_{F}$, hence the observational smallness of $e$ directly puts a limit on $e_{F}$. If $e_{F} \ll e_{R}$, then $\mathbf{e} \simeq \mathbf{e}_{R}$, hence $e_{F} \ll e_{R} \simeq e$. Therefore, a comparable magnitude of $e_{F}$ and $e_{R}$ which cancels them out (for an appropriate $\theta$ ) would present the weakest constraint on $e_{F}$. But even in the unlikely event of complete cancellation, a sizable $\hat{\alpha}_{1}$ cannot hide forever. A finite observational time $T_{\text {obs }}$, hence a finite change in $\theta, \Delta \theta=\dot{\omega}_{\mathrm{PN}} T_{\mathrm{obs}}$, would induce a sizable variation of the eccentricity. We can use this "induced" eccentricity variation to perform the most conservative limit on $\hat{\alpha}_{1}$.

As we can see in the lower panel (a) of figure 5, for a given observational time span, the most conservative configuration is the one in which $\mathbf{e}_{F}$ and $\mathbf{e}_{R}(t)$ cancel out right at the middle of the observational time span, as illustrated in the upper panel (ii). For the most conservative configuration, the "rotating eccentricity" sweeps out an angle $\theta$ from $\pi-\delta$ to $\pi+\delta$ during the observational time span $T_{\text {obs }}$, where $\Delta \theta=\dot{\omega}_{\mathrm{PN}} T_{\text {obs }} \equiv 2 \delta$.

Before moving on, we would like to point out that our analysis accounts for the fact that the timing eccentricity published results from a fit to the whole observational data set of the pulsar binary, spanning 15 years. Hence the published eccentricity (table 1) represents a "weighted" average of a potentially changing eccentricity $e(t)$. After accounting for this, we can get an upper limit for the maximum eccentricity $\bar{e}$ hidden in the data, and from this a limit on $e_{F}$ from (38),

$$
e_{F} \leq \frac{\bar{e}}{\sqrt{1-\sin ^{2} \delta / \delta^{2}}}
$$

which can be converted into an upper limit on $\hat{\alpha}_{1}$ through (37),

$$
\left|\hat{\alpha}_{1}\right|^{\text {upper }}=\frac{1}{\pi^{2}(q-1)} \frac{\bar{e} P_{b}^{2}}{x} \frac{\dot{\omega}_{\mathrm{PN}}}{\sqrt{1-\sin ^{2} \delta / \delta^{2}}}\left(\frac{\sin i}{\sin \psi}\right)\left(\frac{w}{c}\right)^{-1},
$$

where $\psi$ is again the angle between the orbital angular momentum and $\mathbf{w}$ (see figure 1). On the right hand side of (40), for a given $\Omega$, all quantities are observables or can be directly derived from observational quantities, except $\dot{\omega}_{\mathrm{PN}}$. As given in (10), $\dot{\omega}_{\mathrm{PN}}$ is the advance rate of periastron, with potential corrections from the (generalized) EddingtonRobertson-Schiff parameters and PFE parameters. The solution is to take advantages of the smallness of $\delta$, as it is indeed the case in the binaries which we will use to constrain $\hat{\alpha}_{1}$. For PSRs J1012+5307 (and J1738+0333) the $\delta$ is smaller than $10^{\circ}$. When $\delta$ is small, (40) becomes,

$$
\left|\hat{\alpha}_{1}\right|^{\text {upper }} \simeq \frac{2 \sqrt{3}}{\pi^{2}(q-1)} \frac{\bar{e} P_{b}^{2}}{x T_{\mathrm{obs}}}\left(\frac{\sin i}{\sin \psi}\right)\left(\frac{w}{c}\right)^{-1},
$$

where, to first order, $\dot{\omega}_{\mathrm{PN}}$ cancels out in the numerator and denominator, since $\sqrt{1-\sin ^{2} \delta / \delta^{2}} \approx \delta / \sqrt{3}=\dot{\omega} T_{\mathrm{obs}} /(2 \sqrt{3})$. Strictly speaking, the smallness of $\delta$ for PSR J1012+5307 (and PSR J1738+0333) has been inferred from the GR value of $\dot{\omega}_{\mathrm{PN}}$. The argument would break down if there is a factor of a few deviation from GR in these systems. This we consider as unlikely, as there is neither such a deviation from GR in 

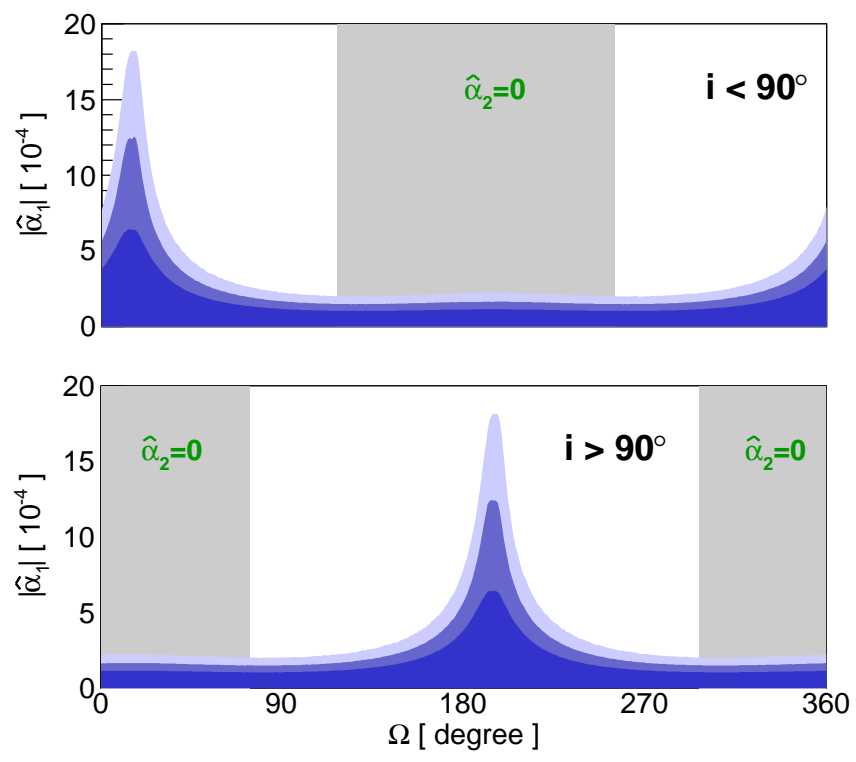

Figure 6. Upper limit of $\left|\hat{\alpha}_{1}\right|$ as a function of the longitude of ascending node $\Omega$, derived from PSR J1012+5307 by using (41) (Upper: $i<90^{\circ}$; Lower: $i>90^{\circ}$ ). The shadowed regions are the allowed values of $\Omega$ at $95 \%$ confidence level, assuming $\hat{\alpha}_{2}=0$ 34. Different contours correspond to $68 \%, 95 \%$, and $99.7 \%$ confidence levels.

the gravitational wave damping of these systems, nor is such a large deviation seen in generic direct tests of $\dot{\omega}$, like in the double pulsar [32].

As mentioned in section 3, PSR J1012+5307 has been observed for 15 years (for the timing solution presented in [34]). During that time, its periastron has already rotated out an angle $\Delta \theta \simeq 10^{\circ}$, which corresponds to $\delta \simeq 5^{\circ}$ in the most conservative configuration. The smallness assumption of $\delta$ is satisfied, hence we can use (41) instead of the more rigorous expression (40).

After properly accounting for all measurement errors, we carry out $10^{5}$ Monte Carlo simulations to get an upper limit of $\hat{\alpha}_{1}$ for every value of $\Omega$ between $0^{\circ}$ and $360^{\circ}$ (in steps of one degree). The results are depicted in figure 6 for $i<90^{\circ}$ (upper) and $i>90^{\circ}$ (lower), respectively. Our calculation uses the worst configuration (see the upper panel (ii) of figure 5), hence the limit is most conservative and reliable. It is easily seen that the results for $i<90^{\circ}$ and $i>90^{\circ}$ are merely shifted by $180^{\circ}$. The existence of the peak near $15^{\circ}$ for the case $i<90^{\circ}\left(195^{\circ}\right.$ for the case $\left.i>90^{\circ}\right)$ is caused by the $1 / \sin \psi$ factor in (40). It can be understood from the $\psi$ curve in the upper panels of figure 2 . It is important that $\sin \psi$ does not vanish for $\Omega \in\left[0^{\circ}, 360^{\circ}\right)$, as this avoids divergencies like in figure 2 for the $\hat{\alpha}_{2}$ test, which would have to be excluded based on probabilistic considerations.

From figure 6, most $\Omega$ realization would limit $\left|\hat{\alpha}_{1}\right|$ to be less than $2 \times 10^{-4}$ (95\% C.L.). Worth to mention that, our confidence level for the $\hat{\alpha}_{1}$ test is purely from measurement errors, in contrast with that from probabilistic assumptions. If we assume a random $\Omega \in\left[0^{\circ}, 360^{\circ}\right)$, a similar constraint is obtained. However, since we want to constrain $\hat{\alpha}_{1}$ 
most robustly, we conservatively adopt the worst configuration $\left(\Omega \simeq 15^{\circ}\right.$ for $i<90^{\circ}$ or $\Omega \simeq 195^{\circ}$ for $i>90^{\circ}$ ) and get a limit

$$
\left|\hat{\alpha}_{1}\right|<1.3 \times 10^{-3} \quad(95 \% \text { C.L. }) .
$$

This limit is one order of magnitude worse than that obtained in [50], see (3). But as it avoids the probabilistic considerations of the method used in [50], we consider this limit as more robust. Furthermore, this limit is more likely to improve in the future than that of [50], which we will discuss when giving the figure of merit of this test, in section 5.3 .

It is worth mentioning that, as we can see in section 2, for small-eccentricity binaries, the effects induced by $\hat{\alpha}_{1}$ and $\hat{\alpha}_{2}$ decouple. Hence this kind of test is not directly influenced by a non-zero $\hat{\alpha}_{2}$. But a vanishing $\hat{\alpha}_{2}$ would tighten the $\hat{\alpha}_{1}$ constraint a little further. If we adopt a zero $\hat{\alpha}_{2}$, or take the Solar limit (2) for $\alpha_{2}$ as a limit for $\hat{\alpha}_{2}$, then the observed $\dot{x}$ of [34] can be attributed totally to the contribution of the proper motion, i.e. (29). Consequently, as mentioned, $\Omega$ can be constrained to certain value ranges [34]. We plot the consistent $\Omega$ values (95\% C.L.) as shadowed regions in figure 6. We can see that, this extra constraint excludes the worst $\Omega$ configuration in both cases of $i<90^{\circ}$ and $i>90^{\circ}$. Hence, with a vanishing $\hat{\alpha}_{2}$, we get a much tighter (conservative) limit of,

$$
\left|\hat{\alpha}_{1}\right|<1.6 \times 10^{-4} \quad(95 \% \text { C.L. }) \text {. }
$$

This limit is comparable to the current best Solar system limit on $\alpha_{1}$, coming from LLR [39], and is only slightly worse than the current best limit (3) for strongly self-gravitating bodies, but based on a method that avoids probabilistic considerations in terms of the exclusion of certain unfavorable angles.

\subsection{PSR J1738+0333, a short orbital period system with a measured eccentricity}

Like PSR J1012+5307, PSR J1738+0333 is also a short orbital period NS-WD binary which can be use to constrain $\hat{\alpha}_{1}$. This binary has been observed for 10 years [20], and during this time, the periastron has advanced by $\Delta \theta \simeq 16^{\circ}$. Unlike PSR J1012+5307, PSR J1738+0333 has a 3- $\sigma$ measurement of the (intrinsic) orbital eccentricity vector (see $\eta$ and $\kappa$ in table 11), while any change in the eccentricity vector is still hidden in the measurement uncertainties. In a PFE test that exploits all the available information about PSR J1738+0333, this fact has to be taken into account (cf. [48], where such a directional information, i.e. the longitude of periastron $\omega$, has been used to improve the constraints on a violation of the strong equivalence principle). But let us first, for simplicity, make only use of the upper limit for the eccentricity (2$\sigma$ upper limit for $e: 5.7 \times 10^{-7}$ ) and apply the method discussed in the previous subsection on PSR J1012+5307, as this method is fast and can be easily compared to the PSR J1012+5307 result, which is based on the same method. For PSR J1738+0333 one finds $\delta \simeq 8^{\circ}$, and furthermore

$$
\left|\hat{\alpha}_{1}\right|<1.6 \times 10^{-4} \quad(95 \% \text { C.L. }) \text {, }
$$



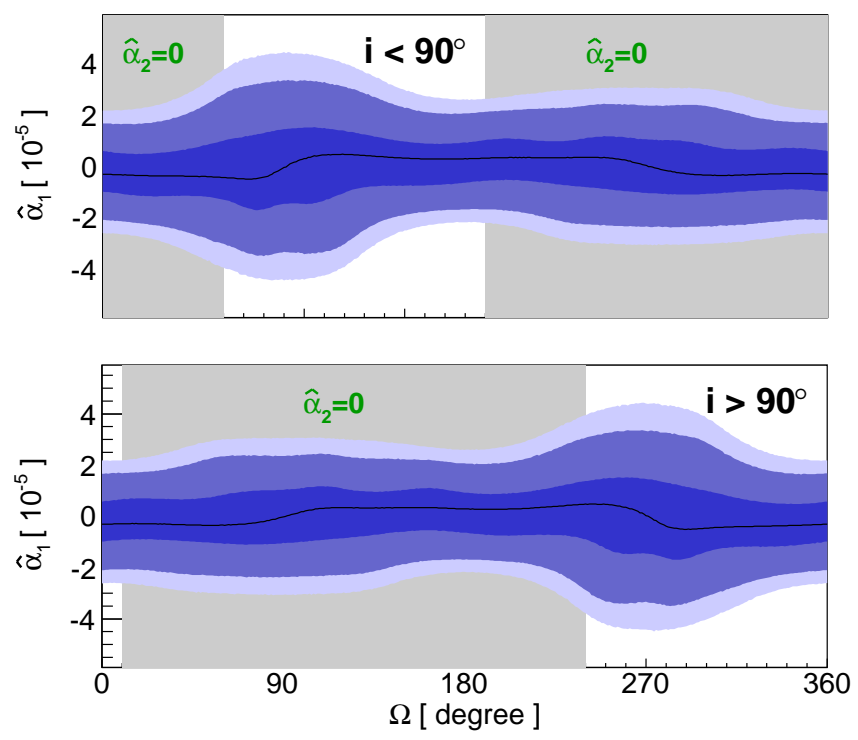

Figure 7. The $\hat{\alpha}_{1}$ constraint from PSR J1738+0333, as a function of the longitude of ascending node $\Omega$ (Upper: $i<90^{\circ}$; Lower: $i>90^{\circ}$ ). The limit is obtained from Monte Carlo simulations, by using full information of the observed eccentricity vector. The shadowed regions are the allowed values of $\Omega$ at $95 \%$ confidence level, assuming $\hat{\alpha}_{2}=0$, see (32). Different contours correspond to $68 \%, 95 \%$, and $99.7 \%$ confidence levels.

for the worst configuration of $\Omega$ and $\theta_{0}$ (i.e. upper panel (ii) of figure 5), without assuming a vanishing $\hat{\alpha}_{2}$. This limit has to be compared to (42). The improvement over that of PSR J1012+5307 comes from the shorter orbital period and a smaller $1 / \sin \psi$ factor for the worst $\Omega$, as can be seen in the upper panels of figure 3 ,

However, for PSR J1738+0333 we have a $3 \sigma$-measurement for the eccentricity vector, instead of just an upper limit on its magnitude (see table 11). The result is expressed in terms of the first and second Laplace-Lagrange parameters, $\eta \equiv e \sin \omega$ and $\kappa \equiv e \cos \omega$ [20], from the ELL1 timing model [33]. Therefore, essentially we have constraints on the magnitude and the direction of the eccentricity vector. To fully include this information, we develop a new method, whose basic idea is depicted in the upper panel (iii) and the lower panel (b) of figure 5, and is to be elaborated below.

We a priori have no knowledge about the magnitudes of $\mathbf{e}_{F}$ and $\mathbf{e}_{R}$, nor the initial angle between them, $\theta_{0}$, at the time when timing observation started. Instead, we have rough information about the superposed eccentricity e, including its magnitude and direction, to $\sim 3 \sigma$ precision (see $\eta$ and $\kappa$ of PSR J1738+0333 in table 1 ), and also the direction of $\mathbf{e}_{F}$ as a function of the unknown $\Omega$. However, we cannot use e directly, for $\eta$ and $\kappa$ were treated as constants when fitting to TOAs, therefore they represent equivalently time-averaged quantities of the observational span. We set up Monte Carlo simulations to select those $\hat{\alpha}_{1}$ which do not conflict with the timing observation of PSR J1738+0333. Our simulation mainly includes the picking of an $\hat{\alpha}_{1}$ (hence $\mathbf{e}_{F}$ ) and $\mathbf{e}_{R}$ (a 
sufficiently large range for $e_{R}>0$ and a $\left.\theta_{0} \in\left[0^{\circ}, 360^{\circ}\right)\right)$, for every $\Omega \in\left[0^{\circ}, 360^{\circ}\right)$. The worst constraint of $\hat{\alpha}_{1}$ is announced as our most conservative limit. All measurement errors are properly considered in our simulations.

The practical implementation to get the constraints for $\hat{\alpha}_{1}$ is as following. First, we randomly choose $\hat{\alpha}_{1}, e_{R}(>0)$, uniformly from wide ranges, and $\theta_{0}$ from $\left[0^{\circ}, 360^{\circ}\right)$, in order to map out a data-cube for these three values. Second, for every point in the data cube we let the eccentricity vector, $\mathbf{e}(t)=\mathbf{e}_{F}+\mathbf{e}_{R}(t)$, evolve under the dynamics given by the Lagrangian for a time span of observations. Then we calculate the time-averaged $\eta$ and $\kappa$, as well as their variances, $\sigma_{\eta}$ and $\sigma_{\kappa}$. We impose the following two criteria to get possible $\hat{\alpha}_{1}, e_{R}, \theta_{0}$ combinations which can still hide in the timing data: 1) The time-averaged $\eta$ and $\kappa$ should agree with observational values within $2 \sigma$ measurement errors (we check that the results are practically unchanged if we use $1 \sigma$ or $3 \sigma$ instead); 2 ) The intrinsic variances of these two parameters, induced by their time evolution, should be smaller than the observational errors, because otherwise, they would contradict the actual measurement of $\eta$ and $\kappa$. By this, we assume that the reported measurement errors are the squared addition of the intrinsic variances and other possible errors, including those possibly from measurement devices and timing models. For each $\Omega$, we accumulate $10^{5}$ events in total, and get a distribution from it. The median values and distribution widths of $\hat{\alpha}_{1}$ are reported in figure 7 for $i<90^{\circ}$ (upper) and $i>90^{\circ}$ (lower), as a function of $\Omega$.

We can see from figure 7 that, $\hat{\alpha}_{1}$ is constrained to the level of $\sim 10^{-5}$. More importantly, in contrast to PSR J1012+5307, the limit only weakly depends on the (presently) unknown angle of the ascending node, $\Omega$. For the worst configurations $\left(\Omega \simeq 92^{\circ}\right.$ for $i<90^{\circ}$ and $\Omega \simeq 273^{\circ}$ for $i>90^{\circ}$ ) one finds

$$
\hat{\alpha}_{1}=-0.4_{-3.1}^{+3.7} \times 10^{-5} \quad(95 \% \text { C.L. }),
$$

which is about 40 times stronger than the limit from PSR J1012+5307 (cf. (42)). As outlined above, this limit is free of any probabilistic considerations related to unknown angles. The limit in (45) is more than five times better than the present best limit on $\alpha_{1}$, coming from LLR [39]. Furthermore, it is also about four times better than the less robust test of [50].

Like in the case of PSR J1012+5307, we could adopt the very small Solar system limit for $\alpha_{2}$ as a limit for $\hat{\alpha}_{2}$, in order to constrain the range of $\Omega$ with the help of $\dot{x}$. But here this would only slightly improve compared to the above constraint, because of the weak dependence on $\Omega$.

It is also instructive to extract from our calculations the possible values of $e_{F}$, in comparison to the observed eccentricity $e$. We find in our Monte Carlo simulations that $e_{F}<1.4 \times 10^{-6}(95 \%$ C.L.) for the most conservative configuration in terms of $i$ and $\Omega$. Compared to the (Shapiro corrected) observed $e=\sqrt{\eta^{2}+\kappa^{2}}=3.4 \times 10^{-7}$, this is only a factor of a few larger. This fact reveals a generic feature of our test, that a too large $e_{F}$ cannot hide in the timing data. It lays down the reasoning of the $\hat{\alpha}_{1}$ test presented here, utilizing long-term pulsar timing of small-eccentricity binaries. 
The method developed here for PSR J1738+0333, is capable of incorporating the directional information of a measured eccentricity vector to constrain $\hat{\alpha}_{1}$. In principle it can also be applied to binary pulsars where no eccentricity is measured, and the direction of the eccentricity vector is unconstrained. For instance, for PSR J1012+5307 this method yields

$$
\hat{\alpha}_{1}=-0.0_{-1.0}^{+1.1} \times 10^{-3} \quad(95 \% \text { C.L. }) .
$$

As expected, this limit is comparable with that of (42), and constitutes a nice test of the implementation used PSR J1738+0333. In general, for systems that do not have a measured eccentricity, the method outline in section 4.1 is preferable, as the method of this subsection is computationally considerably more expensive.

\section{Discussions and summary}

\subsection{Constraints on PFEs from the Galactic frames}

When using the isotropic CMB frame as the assumed preferred frame, we are basically assuming that the preferred frame is determined by the global matter distribution in the Universe, and that the extra vectorial or tensorial components of gravitational interaction are long range, at least comparable to the Hubble radius. While this is generally the most plausible assumption, it is still interesting to consider other, more local preferred frames, like the one related to the rest frame of our Galaxy, or a frame in Galactic co-rotation with the overall local matter at the pulsar's location (cf. [47]). It is straightforward to apply the computations of this paper to these two Galactic frames.

We used the Galactic model of [44], which assumes a distance of $8.0 \mathrm{kpc}$ between the Solar system and the Galactic center, to extract the Galactic rotation curve. Then the velocities of the pulsar binary with respect to the Galactic frame and the local corotating Galactic frame are obtained. They are typically smaller than the velocity with respect to the CMB frame (see table 2). The binary velocity with respect to the local co-rotating frame is about $100 \mathrm{~km} \mathrm{~s}^{-1}$, which is, however, one order of magnitude larger than that of the Solar system 38. Hence, pulsar binaries have the advantage to probe secular PFEs with respect to locally co-rotating Galactic frames, thanks to their peculiar velocity produced by the supernova, while Solar system tests are expected to be clearly less sensitive to such PFEs, because of the small peculiar Solar velocity Table 2 gives, besides the limits for the CMB frame, the limits for the two Galactic frames introduced above, based on the methods presented in sections 3 and 4 . The limits concerning the two Galactic frames are somewhat weaker than that of the CMB frame, because of the smaller peculiar velocities of the pulsar binaries. Nevertheless, they could be of interest for tests of specific gravity theories that might predict or even require (to pass Solar system tests) vector or tensor fields which are aligned with the Galactic or local matter distribution.

『 Nevertheless, see [47] for a constraint of $\alpha_{1}=(1.6 \pm 8.0) \times 10^{-3}$ (95\% C.L.) from LLR when choosing the Barycentric Celestial Reference System as the preferred frame, based on periodic effects. 


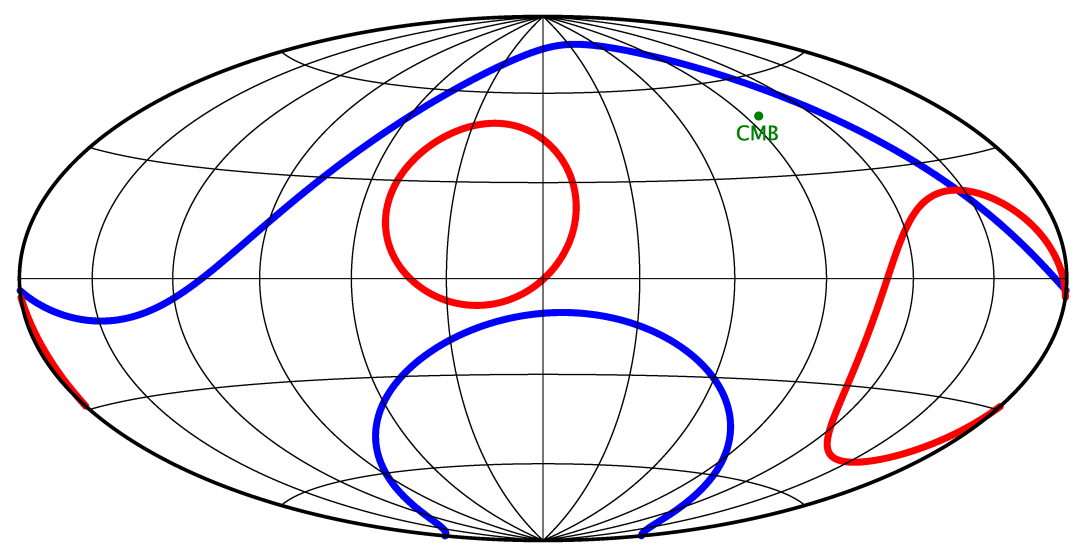

Figure 8. Sky coverage in the $\hat{\alpha}_{1}$ test by PSRs J1012+5307 and J1738+0333. The blue curves mark the directions in the sky where PSR J1012+5307 is insensitive, and the red curves those directions where PSR J1738+0333 is insensitive, due to the unknown $\Omega$. The sky is plotted in an Hammer-Aitoff projection using Galactic coordinates $l$ and $b$. The longitude $l$ increases from right to left, from $l=-180^{\circ}$ to $l=+180^{\circ}$, while the latitude $b$ runs from $b=-90^{\circ}$ to $b=90^{\circ}$ from bottom to top. The grid gives steps of $30^{\circ}$. The plot is based on a $w=369 \mathrm{~km} \mathrm{~s}^{-1}$ for the velocity of the SSB with respect to a potential preferred frame. The label "CMB" denotes the direction of motion with respect to the $\mathrm{CMB}$ frame.

Finally, it is interesting to see the sky coverage of the two binary pulsars in terms of a sensitivity towards a preferred frame. In the spirit of a "PFE pulsar antenna array", proposed in [51], two suitable binary pulsars can probe for a preferred reference frame in (almost) any direction in the sky. Figure 8 shows the combined sky coverage in the $\hat{\alpha}_{1}$ test for PSRs J1012+5307 and J1738+0333, for $w=369 \mathrm{~km} \mathrm{~s}^{-1}$, the same magnitude as the velocity of the SSB with respect to the CMB. The figure nicely illustrates why PSR J1738+0333 is more suitable to test the CMB frame, since the "CMB" dot is far away from any of the red curves, meaning that the CMB direction lies in the area where the system is particularly sensitive. The absence of any divergencies in figure $\mathbf{7}$ is a consequence of this.

\subsection{Strong-field modifications}

When comparing the results of this paper, obtained from pulsar-WD systems, with Solar system experiments, one has to keep in mind that alternative gravitational theories, in general, are expected to predict strong-field modifications of the PPN parameters due to the strong internal gravitational field of the pulsar. As an example, in scalar-tensor gravity the PPN parameter $\gamma$ generalizes to

$$
\hat{\gamma} \equiv \gamma_{A B}=1-\frac{2 \alpha_{A} \alpha_{B}}{1+\alpha_{A} \alpha_{B}},
$$

for a binary pulsar system, where $\alpha_{A}$ and $\alpha_{B}$ are the effective scalar coupling constants of pulsar and companion, respectively [13]. The weak-field PPN parameter $\gamma$ is recovered 
for $\alpha_{A}=\alpha_{B}=\alpha_{0}$. In GR one has $\hat{\gamma}=\gamma=1$. In the strong-field regime of a NS, $\hat{\gamma}$ can deviate significantly from $\gamma$ due to strong-field scalarization effects [14]. Similarly, we may expect that $\hat{\alpha}_{1}$ and $\hat{\alpha}_{2}$ deviate from their PPN correspondents, $\alpha_{1}$ and $\alpha_{2}$. In the absence of non-perturbative effects, one can illustrate this as an expansion in the compactnesses $c_{A}$ and $c_{B}$ of the bodies [15]. In our case, we would write something like

$$
\hat{\alpha}_{a}=\alpha_{a}+\mathcal{K}_{i} c_{i}+\mathcal{K}_{i j} c_{i} c_{j}+\ldots,
$$

where $a=1,2$ and $\mathcal{K}_{i}$ and $\mathcal{K}_{i j}$ are coefficients characterizing deviations from general relativity, and $c_{i} \sim G m_{i} / R_{i} c^{2}$ with mass $m_{i}$ and radius $R_{i}$ of body $i$. The compactnesses for the Earth and the Sun are roughly $c_{\oplus} \sim 10^{-10}$ and $c_{\odot} \sim 10^{-6}$, respectively, which suppress $\mathcal{K}_{i^{-}}$and $\mathcal{K}_{i j}$-related physical effects dramatically. In contrast, NSs have $c_{\mathrm{NS}} \sim 0.2$, which is one of the reasons why pulsar timing experiments are ideal probes for gravity effects associated with strong gravitational fields. Consequently, in a NS-WD system $\left(c_{\mathrm{WD}} \sim 10^{-4}\right)$ we could still have a significant $\hat{\alpha}_{a}$, even if there is a tight Solar system constraint for $\alpha_{a}$. This, for instance, supports the importance of the $\hat{\alpha}_{2}$ limits obtained in this paper.

Finally, when discussing the constraints on parameters of alternative gravity theories, one should be aware of a potential compactness-dependent (or massdependent) nature of these parameters. Especially when combining different systems, like the $\hat{\alpha}_{1}$ test in [50], or the combined probability distribution function of the $\hat{\alpha}_{2}$ test presented in this paper (black solid histogram of figure 4). Such tests implicitly assume that the parameter is approximately the same for all systems under investigation. We are aware of this potential problem in our calculation. In the case of the PSRs J1012+5307 and J1738+0333 binary systems one can argue that the similarity in the masses justifies such an assumption. However, in the presence of phenomena related to some critical mass, like the spontaneous scalarization discovered in [14], even a small difference in masses does not allow such an assumption. Our proposed robust test for $\hat{\alpha}_{1}$ overcomes this "mass-dependence" problem by only considering one system. Hence our final results on $\hat{\alpha}_{1}$ are more suitable to be quoted along with mentioning the specific system were it was obtained from and the related neutron-star mass.

\subsection{Figures of merit and further potential improvements}

Finally, let us discuss potential improvements of the current limits of $\hat{\alpha}_{1}$ and $\hat{\alpha}_{2}$. For this, one conveniently identifies the figures of merit for the different tests. Besides details related to the shape and the size of the orbit, various geometrical angles and the barycentric velocity with respect to the preferred frame (w) play a role in our tests. Therefore, in principle our figures of merit would depend on the geometrical configuration of binary systems under consideration, as well as their proper motions with respect to the preferred frame. However, after we drop the geometrical dependencies and ignore potential difference in $\mathbf{w}$, we can roughly get a figure of merit of our tests.

As for the $\hat{\alpha}_{1}$ test, the traditional method has a figure of merit, $P_{b}^{1 / 3} / e$ [12], which means that the strength of this test is not to improve until new systems with 
higher $P_{b}^{1 / 3} / e$ are discovered. In contrast, our figure of merit for the robust $\hat{\alpha}_{1}$ test is $T_{\text {obs }} /\left(P_{b}^{4 / 3} \bar{e}\right)$ for binary pulsars with unmeasured eccentricities $e<\bar{e}$ (cf. (41)) ). Besides the discoveries of new systems with smaller $P_{b}^{4 / 3} \bar{e}$, the constraint has the potential to improve when the observational span becomes longer. In fact, it improves as $T_{\text {obs }}^{3 / 2}$, as long as e remains smaller than $\bar{e}$, and $\dot{\omega} T_{\text {obs }} \lesssim 1$. For a binary pulsar with measured eccentricity $e$, we get a similar figure, where $\bar{e}$ is to be replaced with $\sigma_{e}$, the measurement error of the eccentricity vector, or that of the first and second LaplaceLagrange parameters, namely $\sigma_{\eta}$ and $\sigma_{\kappa}$. If in the future the secular evolution of the eccentricity vector in PSR J1738+0333, due to the relativistic periastron advance, can be measured, we could further constrain a potential polarization of the orbit caused by a non-vanishing $\hat{\alpha}_{1}$, or even detect the presence of a significant PFE eccentricity $\mathbf{e}_{F}$.

In contrast to our secular effects, the limit from LLR is based on periodic effects (see e.g. [17]), and therefore only improves as $T_{\text {obs }}^{1 / 2}$. Moreover, to test PFEs in LLR one has to deal with the motion of the Earth-Moon system around the Sun, introducing tidal forces and an annually changing $\mathbf{w}$, hence leading to the theoretical complexity of a three-body problem in the presence of a preferred frame [17].

For the $\hat{\alpha}_{2}$ test one finds the figure of merit to be $1 /\left(P_{b}^{1 / 3} \sigma_{\dot{x}}\right)$ from (25), where $\sigma_{\dot{x}}$ is the measurement uncertainty of $\dot{x}$. Hence, more relativistic systems (smaller $P_{b}$ ) with high timing precision (especially of $\dot{x}$ ) are advantageous to do the $\hat{\alpha}_{2}$ test. For the systems discussed here, namely PSRs J1012+5307 and J1738+0333, persistent timing observations will reduce the measurement uncertainty of $\sigma_{\dot{x}}$. Hence the $\hat{\alpha}_{2}$ test will improve continuously as $T_{\text {obs }}^{3 / 2}$, in contrast with that of [42]. An even faster improvement is expected to come from new receiver and backend technologies and new telescopes.

It is worth noting that a measurement of the unknown longitude of the ascending node, $\Omega$, would improve both the $\hat{\alpha}_{1}$ and the $\hat{\alpha}_{2}$ test. If $\Omega$ can be determined independently, even rough constraints on $\Omega$ would make these tests more efficient. It would eliminate the (systematical) "double peak" structure in the probability distribution function of $\hat{\alpha}_{2}$ in figure 4. Also it would select a specific limit on $\hat{\alpha}_{1}$ from figures 6 or 7 , where presently we are conservatively using the worst $\Omega$ configuration. Unfortunately, neither pulsar timing nor optical astrometry are likely to provide such a measurement in the near future. Maybe scintillation measurements would be able to provide interesting constraints on $\Omega$, like this is the case for the double pulsar (Rickett et al, in prep.).

\subsection{A brief summary of the results}

In summary, we presented an extended orbital dynamics of pulsar binaries under the influence of preferred frame effects that accounts for both generalized PPN parameters, $\hat{\alpha}_{1}$ and $\hat{\alpha}_{2}$. In the limit of a small eccentricity, orbital effects from $\hat{\alpha}_{1}$ and $\hat{\alpha}_{2}$ decouple. We implemented two new methods to constrain $\hat{\alpha}_{1}$ and $\hat{\alpha}_{2}$, by directly constraining secular orbital changes expected from a violation of local Lorentz invariance in the gravitational sector. Both methods have been applied to the two binary pulsars PSRs 
J1012+5307 and J1738+0333, where we have full spatial velocity information. For a frame at rest with respect to the CMB, the best limit we obtain is

$$
\hat{\alpha}_{1}=-0.4_{-3.1}^{+3.7} \times 10^{-5} \quad(95 \% \text { C.L. }),
$$

which avoids the probabilistic considerations of previous methods, and clearly surpasses the current best limits obtained with both, weakly (Solar system) and strongly (binary pulsars) self-gravitating bodies, namely (11) and (3).

Concerning $\hat{\alpha}_{2}$, the best limit we obtain is

$$
\left|\hat{\alpha}_{2}\right|<1.8 \times 10^{-4} \quad(95 \% \text { C.L. }) .
$$

This limit is still three orders of magnitude weaker than the weak-field limit obtained in the Solar system, but constrains possible deviations related to the strong internal gravitational fields of NSs. The limit here surpasses the current best limit for strongly self-gravitating bodies, namely (4), by three orders of magnitude, although strictly speaking they are different in their physical nature, as (4) probes the interaction between two strongly self-gravitating bodies, in contrast to the pulsar-WD systems used in this paper. A drawback of the $\hat{\alpha}_{2}$ limit presented here is that it is still based on probabilistic considerations in excluding unfavorable values of the longitude of the ascending node $\Omega$, and the combination of two systems with different NS masses.

Our new methods promise continuous improvements with on-going timing observations of known systems, as well as the discovery of new suitable systems. In addition, new receiver and backend technologies as well as new telescopes, like FAST [40] and SKA [45], will tremendously improve these tests.

\section{Acknowledgments}

We thank John Antoniadis, William Coles, Paulo Freire, Michael Kramer and Clifford Will for stimulating discussions. We are grateful to Michael Kramer for carefully reading the manuscript. Lijing Shao is supported by China Scholarship Council (CSC).

\section{References}

[1] J. Antoniadis, M. H. van Kerkwijk, D. Koester, P. C. C. Freire, N. Wex, T. M. Tauris, M. Kramer, and C. G. Bassa. The relativistic pulsar-white dwarf binary PSR J1738+0333 I. Mass determination and evolutionary history. MNRAS, 423:3316, 2012.

[2] Z. Arzoumanian, K. Joshi, F. A. Rasio, and S. E. Thorsett. Orbital parameters of the PSR B1620-26 triple system. In S. Johnston, M. A. Walker, \& M. Bailes, editor, IAU Colloquium 160 - Pulsars: Problems and Progress, Astronomical Society of the Pacific Conference Series, 105:525, 1996.

[3] Q. G. Bailey and V. A. Kostelecký. Signals for Lorentz violation in post-Newtonian gravity. Phys. Rev. D, 74:045001, 2006.

[4] B. M. Barker and R. F. O'Connell. Relativistic effects in the binary pulsar PSR 1913+16. ApJL, 199:L25, 1975.

[5] J. D. Bekenstein. Relativistic gravitation theory for the modified Newtonian dynamics paradigm. Phys. Rev. D, 70:083509, 2004 [Erratum: Phys. Rev. D, 71:069901, 2005]. 
[6] J. F. Bell, F. Camilo, and T. Damour. A tighter test of the local Lorentz invariance of gravity using PSR J2317+1439. ApJ, 464:857, 1996.

[7] M. Burgay, N. D'Amico, A. Possenti, R. N. Manchester, A. G. Lyne, B. C. Joshi, M. A. McLaughlin, M. Kramer, J. M. Sarkissian, F. Camilo, V. Kalogera, C. Kim, and D. R. Lorimer. An increased estimate of the merger rate of double neutron stars from observations of a highly relativistic system. Nature, 426:531, 2003.

[8] P. J. Callanan, P. M. Garnavich, and D. Koester. The mass of the neutron star in the binary millisecond pulsar PSR J1012+5307. MNRAS, 298:207, 1998.

[9] S. Chandrasekhar and G. Contopoulos. On a post-Galilean transformation appropriate to the post-Newtonian theory of Einstein, Infeld and Hoffmann. Royal Society of London Proceedings Series A, 298:123, 1967.

[10] T. Damour and N. Deruelle. General relativistic celestial mechanics of binary systems. II. The post-Newtonian timing formula. Ann. Inst. Henri Poincaré Phys. Théor., 44:263, 1986.

[11] T. Damour and G. Schäfer. New tests of the strong equivalence principle using binary-pulsar data. Physical Review Letters, 66:2549, 1991.

[12] T. Damour and G. Esposito-Farèse. Testing local Lorentz invariance of gravity with binary-pulsar data. Phys. Rev. D, 46:4128, 1992.

[13] T. Damour and G. Esposito-Farèse. Tensor-multi-scalar theories of gravitation. Class. Quantum Grav., 9:2093, 1992.

[14] T. Damour and G. Esposito-Farèse. Nonperturbative strong-field effects in tensor-scalar theories of gravitation. Physical Review Letters, 70:2220, 1993.

[15] T. Damour and G. Esposito-Farèse. Testing gravity to second post-Newtonian order: A fieldtheory approach. Phys. Rev. D, 53:5541, 1996.

[16] T. Damour and J. H. Taylor. On the orbital period change of the binary pulsar PSR 1913+16. ApJ, 366:501, 1991.

[17] T. Damour and D. Vokrouhlický. Testing for gravitationally preferred directions using the lunar orbit. Phys. Rev. D, 53:6740, 1996.

[18] T. Damour and J. H. Taylor. Strong-field tests of relativistic gravity and binary pulsars. Phys. Rev. D, 45:1840, 1992.

[19] B. Z. Foster. Strong field effects on binary systems in Einstein-aether theory. Phys. Rev. D, 76:084033, 2007.

[20] P. C. C. Freire, N. Wex, G. Esposito-Farèse, J. P. W. Verbiest, M. Bailes, B. A. Jacoby, M. Kramer, I. H. Stairs, J. Antoniadis, and G. H. Janssen. The relativistic pulsar-white dwarf binary PSR J1738+0333 II. The most stringent test of scalar-tensor gravity. MNRAS, 423:3328, 2012.

[21] G. Hinshaw, J. L. Weiland, R. S. Hill, and et al. Five-year Wilkinson Microwave Anisotropy Probe observations: data processing, sky maps, and basic results. ApJS, 180:225, 2009.

[22] T. Jacobson and D. Mattingly. Gravity with a dynamical preferred frame. Phys. Rev. D, 64:024028, 2001.

[23] B. A. Jacoby. Recycled pulsars. PhD thesis, California Institute of Technology, California, USA, 2005.

[24] N. Jarosik, C. L. Bennett, J. Dunkley, and et al. Seven-year Wilkinson Microwave Anisotropy Probe (WMAP) observations: sky maps, systematic errors, and basic results. ApJS, 192:14, 2011.

[25] K. J. Joshi and F. A. Rasio, Distant companions and planets around millisecond pulsars. ApJ, 488:901, 1997.

[26] V. M. Kaspi, M. Bailes, R. N. Manchester, B. W. Stappers, and J. F. Bell. Evidence from a processing pulsar orbit for a neutron-star birth kick. Nature, 381:584, 1996.

[27] V. M. Kaspi, A. G. Lyne, R. N. Manchester, F. Crawford, F. Camilo, J. F. Bell, N. D'Amico, I. H. Stairs, N. P. F. McKay, D. J. Morris, and A. Possenti. Discovery of a young radio pulsar in a relativistic binary orbit. ApJ, 543:321, 2000.

[28] S. M. Kopeikin On possible implications of orbital parallaxes of wide orbit binary pulsars and 
their measurability. ApJL, 439:L5, 1995.

[29] S. M. Kopeikin. Proper motion of binary pulsars as a source of secular variations of orbital parameters. ApJL, 467:L93, 1996.

[30] V. A. Kostelecký and N. Russell. Data tables for Lorentz and CPT violation. Reviews of Modern Physics, 83:11, 2011.

[31] M. Kramer, I. H. Stairs, R. N. Manchester, M. A. McLaughlin, A. G. Lyne, R. D. Ferdman, M. Burgay, D. R. Lorimer, A. Possenti, N. D'Amico, J. M. Sarkissian, G. B. Hobbs, J. E. Reynolds, P. C. C. Freire, and F. Camilo. Tests of general relativity from timing the double pulsar. Science, 314:97, 2006.

[32] M. Kramer and N. Wex. The double pulsar system: a unique laboratory for gravity. Class. Quantum Grav., 26:073001, 2009.

[33] C. Lange, F. Camilo, N. Wex, M. Kramer, D. C. Backer, A. G. Lyne, and O. Doroshenko. Precision timing measurements of PSR J1012+5307. MNRAS, 326:274, 2001.

[34] K. Lazaridis, N. Wex, A. Jessner, M. Kramer, B. W. Stappers, G. H. Janssen, G. Desvignes, M. B. Purver, I. Cognard, G. Theureau, A. G. Lyne, C. A. Jordan, and J. A. Zensus. Generic tests of the existence of the gravitational dipole radiation and the variation of the gravitational constant. MNRAS, 400:805, 2009.

[35] D. R. Lorimer and M. Kramer. Handbook of Pulsar Astronomy. Cambridge University Press, 2004.

[36] D. R. Lorimer, A. G. Lyne, L. Festin, and L. Nicastro. Birth rate of millisecond pulsars. Nature, 376:393, 1995.

[37] A. G. Lyne, M. Burgay, M. Kramer, A. Possenti, R. N. Manchester, F. Camilo, M. A. McLaughlin, D. R. Lorimer, N. D’Amico, B. C. Joshi, J. Reynolds, and P. C. C. Freire. A double-pulsar system: a rare laboratory for relativistic gravity and plasma physics. Science, 303:1153, 2004.

[38] F. Mignard. Local galactic kinematics from Hipparcos proper motions. Astron. Astroph., 354:522, 2000.

[39] J. Müller, J. G. Williams, and S. G. Turyshev. Lunar laser ranging contributions to relativity and geodesy. In H. Dittus, C. Lammerzahl, \& S. G. Turyshev, editor, Lasers, Clocks and Drag-Free Control: Exploration of Relativistic Gravity in Space, Astrophysics and Space Science Library, 349:457, 2008.

[40] R. Nan, D. Li, C. Jin, Q. Wang, L. Zhu, W. Zhu, H. Zhang, Y. Yue, and L. Qian. The five-hundred aperture spherical radio telescope (FAST) project. Int. J. Mod. Physics D, 20:989, 2011.

[41] L. Nicastro, A. G. Lyne, D. R. Lorimer, P. A. Harrison, M. Bailes, and B. D. Skidmore. PSR J1012+5307 - a 5.26-millisecond pulsar in a 14.5-hour binary system. MNRAS, 273:L68, 1995.

[42] K. Nordtvedt. Probing gravity to the second post-Newtonian order and to one part in $10^{7}$ using the spin axis of the sun. ApJ, 320:871, 1987.

[43] K. Nordtvedt and C. M. Will. Conservation laws and preferred frames in relativistic gravity. II. Experimental evidence to rule out preferred-frame theories of gravity. ApJ, 177:775, 1972.

[44] B. Paczynski. A test of the galactic origin of gamma-ray bursts. ApJ, 348:485, 1990.

[45] R. Smits, M. Kramer, B. Stappers, D. R. Lorimer, J. Cordes, and A. Faulkner. Pulsar searches and timing with the square kilometre array. Astron. Astroph., 493:1161, 2009.

[46] I. S. Shklovskii. Possible causes of the secular increase in pulsar periods. Soviet Ast., 13:562, 1970 .

[47] M. Soffel, S. Klioner, J. Müller, and L. Biskupek. Gravitomagnetism and lunar laser ranging. Phys. Rev. D, 78:024033, 2008.

[48] I. H. Stairs, A. J. Faulkner, A. G. Lyne, M. Kramer, D. R. Lorimer, M. A. McLaughlin, R. N. Manchester, G. B. Hobbs, F. Camilo, A. Possenti, M. Burgay, N. D’Amico, P. C. Freire, and P. C. Gregory. Discovery of three wide-orbit binary pulsars: implications for binary evolution and equivalence principles. ApJ, 632:1060, 2005.

[49] N. Wex. A timing formula for main-sequence star binary pulsars. MNRAS, 298:67, 1998.

[50] N. Wex. Small-eccentricity binary pulsars and relativistic gravity. In M. Kramer, N. Wex, \& 
R. Wielebinski, editor, IAU Colloq. 177: Pulsar Astronomy - 2000 and Beyond, Astronomical Society of the Pacific Conference Series, 202:113, 2000.

[51] N. Wex and M. Kramer. A characteristic observable signature of preferred-frame effects in relativistic binary pulsars. MNRAS, 380:455, 2007.

[52] C. M. Will. Theory and Experiment in Gravitational Physics. Cambridge University Press, 1993.

[53] C. M. Will. The confrontation between general relativity and experiment. Living Rev. Relativity, 9:3, 2006 [URL (cited on 2012-07-22): http://www.livingreviews.org/lrr-2006-3.

[54] C. M. Will and K. Nordtvedt. Conservation laws and preferred frames in relativistic gravity. I. Preferred-frame theories and an extended PPN formalism. ApJ, 177:757, 1972. 
Table 1. Relevant parameters in the PFE calculations for PSRs J1012+5307 [34, 33] and J1738+0333 [20, 1].

\begin{tabular}{|c|c|c|}
\hline Pulsars & PSR J1012+5307 & PSR J1738+0333 \\
\hline Right Ascension, $\alpha$ (J2000) & $10^{\mathrm{h}} 12^{\mathrm{m}} 33.4341010(99)$ & $17^{\mathrm{h}} 38^{\mathrm{m}} 53.9658386(7)$ \\
\hline Declination, $\delta(\mathrm{J} 2000)$ & $53^{\circ} 07^{\prime} 02^{\prime \prime} .60070(13)$ & $03^{\circ} 33^{\prime} 10^{\prime \prime} .86667(3)$ \\
\hline Proper motion in $\alpha, \mu_{\alpha}\left(\operatorname{mas~yr}^{-1}\right)$ & $2.562(14)$ & $7.037(5)$ \\
\hline Proper motion in $\delta, \mu_{\delta}\left(\operatorname{mas~yr}^{-1}\right)$ & $-25.61(2)$ & $5.073(12)$ \\
\hline Distance, $d(\mathrm{kpc})$ & $0.836(80)$ & $1.47(10)$ \\
\hline Spin period, $P(\mathrm{~ms})$ & $5.255749014115410(15)$ & $5.850095859775683(5)$ \\
\hline Orbital period, $P_{b}(\mathrm{~d})$ & $0.60467271355(3)$ & $0.3547907398724(13)$ \\
\hline Projected semi-major axis, $x$ (lt-s) & $0.5818172(2)$ & $0.343429130(17)$ \\
\hline$\eta \equiv e \sin \omega\left(10^{-7}\right)^{\mathrm{a}}$ & $-1.4 \pm 3.4$ & $-1.4 \pm 1.1$ \\
\hline$\kappa \equiv e \cos \omega\left(10^{-7}\right)$ & $0.6 \pm 3.1$ & $3.1 \pm 1.1$ \\
\hline Time derivative of $x, \dot{x}\left(10^{-15} \mathrm{~s} \mathrm{~s}^{-1}\right)$ & $2.3(8)$ & $0.7(5)$ \\
\hline Time derivative of $P_{b}, \dot{P}_{b}\left(10^{-15} \mathrm{ss}^{-1}\right)$ & $50(14)$ & $-17.0(3.1)$ \\
\hline Radial velocity, $v_{r}\left(\mathrm{~km} \mathrm{~s}^{-1}\right)$ & $44(8)$ & $-42(16)$ \\
\hline Mass ratio, $q \equiv m_{p} / m_{c}$ & $10.5(5)$ & $8.1(2)$ \\
\hline $\mathrm{WD}$ mass, $m_{c}\left(\mathrm{M}_{\odot}\right)$ & $0.16(2)$ & $0.181_{-0.007}^{+0.008}$ \\
\hline Pulsar mass, $m_{p}\left(\mathrm{M}_{\odot}\right)$ & $1.64(22)$ & $1.46_{-0.05}^{+0.06}$ \\
\hline Mass function, $f\left(\mathrm{M}_{\odot}\right)$ & 0.000578 & 0.0003455 \\
\hline Inclination, $i$ (deg) & $52(4)$ or $128(4)$ & $32.6(10)$ or $147.4(10)$ \\
\hline Advance of periastron (in GR), $\dot{\omega}_{\mathrm{PN}}^{(\mathrm{GR})}\left(\mathrm{deg} \mathrm{yr}^{-1}\right)$ & $0.69(6)$ & $1.57(5)$ \\
\hline "Characteristic" velocity (in GR), $\mathcal{V}_{O}^{(\mathrm{GR})}\left(\mathrm{km} \mathrm{s}^{-1}\right)$ & $308(13)$ & $355(5)$ \\
\hline
\end{tabular}


Table 2. Limits on the PFE parameters $\hat{\alpha}_{1}$ and $\hat{\alpha}_{2}$ at $95 \%$ confidence level, from the NS-WD binaries, PSRs J1012+5307 and J1738+0333 (see text for details).

\begin{tabular}{llccc}
\hline Pulsar Binary & Preferred Frame & $w\left(\mathrm{~km} \mathrm{~s}^{-1}\right)$ & $\hat{\alpha}_{1}$ & $\hat{\alpha}_{2}$ \\
\hline \multirow{5}{*}{ J1012+5307 } & CMB & $477(14)$ & $\left|\hat{\alpha}_{1}\right|<1.3 \times 10^{-3}$ & $\left|\hat{\alpha}_{2}\right|<3.6 \times 10^{-4}$ \\
& Galaxy & $157(15)$ & $\left|\hat{\alpha}_{1}\right|<8.3 \times 10^{-3}$ & $\left|\hat{\alpha}_{2}\right|<7.9 \times 10^{-3}$ \\
& Local Galactic rotation & $114(23)$ & $\left|\hat{\alpha}_{1}\right|<7.5 \times 10^{-3}$ & $\left|\hat{\alpha}_{2}\right|<1.1 \times 10^{-2}$ \\
\hline \multirow{5}{*}{ J1738+0333 } & CMB & $327(6)$ & $\hat{\alpha}_{1}=-0.4_{-3.1}^{+3.7} \times 10^{-5}$ & $\left|\hat{\alpha}_{2}\right|<2.9 \times 10^{-4}$ \\
& Galaxy & $265(6)$ & $\hat{\alpha}_{1}=-0.3_{-4.0}^{+4.5} \times 10^{-5}$ & $\left|\hat{\alpha}_{2}\right|<8.3 \times 10^{-4}$ \\
& Local Galactic rotation & $82(10)$ & $\hat{\alpha}_{1}=+0.1_{-4.1}^{+4.2} \times 10^{-4}$ & $\left|\hat{\alpha}_{2}\right|<1.0 \times 10^{-2}$ \\
\hline \multirow{5}{*}{ Combined } & CMB & - & - & $\left|\hat{\alpha}_{2}\right|<1.8 \times 10^{-4}$ \\
& Galaxy & - & - & $\left|\hat{\alpha}_{2}\right|<4.5 \times 10^{-4}$ \\
& Local Galactic rotation & - & - & $\left|\hat{\alpha}_{2}\right|<3.4 \times 10^{-3}$ \\
\hline
\end{tabular}

